\title{
Crystal structures of the recombinant $\beta$-Factor XIIa protease with bound Thr-Arg and Pro-Arg substrate mimetics
}

monika pathak*, rosa manna, chan li, bubacarr kaira, Badraldin Kareem, Benny Belviso, camila Bonturi, ingrid dreveny, peter fischer, Lodewijk dekker, Maria oliva and jonas emsley

CONFIDENTIAL - NOT TO BE REPRODUCED, QUOTED NOR SHOWN TO OTHERS

SCIENTIFIC MANUSCRIPT

For review only.

Monday 13 May 2019

Category: research papers

Co-editor:

Professor R. Garratt

Telephone: +55 $1633739874 / 9846$

Fax: +55 1633739881

Email: richard@ifsc.usp.br

Contact author:

jonas emsley

School of Pharmacy, university of nottingham, university of nottingham, nottingham, Nottingham, ng72rd, United Kingdom

Telephone: 01158467092

Fax: 01158468002

Email: jonas.emsley@nottingham.ac.uk 


\title{
Crystal structures of the recombinant $\beta$-Factor XIla protease with bound Thr-Arg and Pro-Arg substrate mimetics
}

\author{
Monika Pathak ${ }^{1 *}$, Rosa Manna ${ }^{1 *}$, Chan $\mathrm{Li}^{1}$, Bubacarr G Kaira ${ }^{1}$, Badraldin Kareem \\ Hamad $^{1}$, Benny Danilo Belviso ${ }^{1}$, Camila R Bonturi ${ }^{2}$, Ingrid Dreveny ${ }^{1}$, Peter M \\ Fischer $^{1}$, Lodewijk V Dekker ${ }^{1}$, Maria Luiza Viela Oliva ${ }^{2}$, and Jonas Emsley ${ }^{1 \pi}$. \\ ${ }^{1}$ Centre for Biomolecular Sciences, School of Pharmacy, University of Nottingham, UK. \\ ${ }^{2}$ Biochemistry Department, Federal University of São Paulo, 04044-020, São Paulo - SP, Brazil
}

"To whom correspondence should be addressed: Jonas Emsley, School of Pharmacy, Centre for Biomolecular Sciences, University of Nottingham, Nottingham NG7 2RD, United Kingdom; jonas.emsley@nottingham.ac.uk; Tel.(+44) 115-8467092; Fax. (+44) 115- 8468002 *joint first authors.

Synopsis: Coagulation factor XII (FXII) is a key initiator of the contact pathway and kinin generation. We report the first peptido-mimetic complex structure for the activated protease domain $\beta$ FXIIa. These crystal structures of $\beta$ FXIIa provide insight into serine protease substrate and inhibitor recognition. 


\section{Abstract}

Coagulation factor XII (FXII) is a key initiator of the contact pathway which contributes to inflammatory pathways. FXII circulates as a zymogen, which when auto-activated forms factor XIIa (FXIIa). We report production of recombinant FXIIa protease domain ( $\beta$ FXIIa ${ }^{\mathrm{His}}$ ) with yields of $\sim 1-2 \mathrm{mg} / \mathrm{L}$ of insect cell culture. A second construct utilised an N-terminal maltose-binding protein (MBP) fusion (MBP- $\beta$ FXIIa $\left.{ }^{\mathrm{His}}\right)$. Crystal structures were determined for MBP-BFXIIa ${ }^{\text {His }}$ in complex with inhibitor D-Phe-Pro-Arg chloromethyl ketone (PPACK) and $\beta \mathrm{FXIIa}^{\mathrm{His}}$ in isolation. The $\beta \mathrm{FXIIa}^{\mathrm{His}}$ structure revealed the S2 and S1 pockets were occupied with Thr and Arg residues, respectively, from an adjacent molecule in the crystal. The Thr-Arg sequence mimics the P2-P1 FXIIa cleavage site residues present in the natural substrates prekallikrein and FXII and Pro-Arg (from PPACK) mimics the Factor XI cleavage site. A comparison of the $\beta \mathrm{FXIIa}^{\mathrm{His}}$ structure with the available crystal structure of the zymogen-like FXII protease revealed large conformational changes centred around the S1 pocket and an alternate conformation for the 99-loop, Tyr99 and the S2 pocket. Further comparison with activated protease structures for factors IXa and Xa, which also have residue Tyr99, reveals that a more open form of the S2 pocket only occurs in the presence of a substrate mimetic. FXIIa inhibitors EctTI and Infestin-4 have Pro-Arg and Phe-Arg P2-P1 sequences respectively and we also describe the interactions these inhibitors form with $\beta$ FXIIa. These structural studies of $\beta$ FXIIa provide insight into substrate and inhibitor recognition and establish a scaffold for structure-guided drug design of novel anti-thrombotic and anti-inflammatory agents.

Keywords: coagulation factor XII; serine protease; crystal structure; inhibitor complex 


\section{Introduction}

The contact activation system is initiated via auto-activation of the serine protease coagulation factor XII (FXII) (Weidmann et al., 2017). Activated FXII (FXIIa) cleaves substrates coagulation factor XI (FXI), to initiate the intrinsic pathway of coagulation, and prekallikrein (PK), which results in the cleavage of kininogen and bradykinin (BK) formation (Maas \& Renne, 2018). A number of studies have established that inhibition of FXII activity reduces the formation of pathological thrombi without compromising physiological haemostasis in both murine and primate models of cardiovascular disease (Renne et al., 2012, Kleinschnitz et al., 2006, Matafonov et al., 2014). FXII has also been implicated in inflammatory pathways (Hess et al., 2017, Bender et al., 2017) and hereditary angioedema via identification of a gain-of-function mutation in the $F 12$ gene (encodes FXII), which results in excessive formation of BK (Cichon et al., 2006).

Natural inhibitors of the FXII protease in humans include the serpin C1INH (Nickel et al., 2017) and histidine rich glycoprotein (HRG) (MacQuarrie et al., 2011). FXII inhibitors from exogenous sources have been described including corn trypsin inhibitor (CTI) (Mahoney et al., 1984), Enterolobium contortisiliquum trypsin inhibitor (EcTI) (Batista et al., 1996), Triatoma infestans infestin-4 (Campos et al., 2004) and Escherichia coli ecotin (Ulmer et al., 1995). CTI is widely used as an inhibitor that is selective for FXIIa and the intrinsic pathway of coagulation, but does not affect the extrinsic pathway. In vivo studies in animal models of thrombosis demonstrate infestin-4 provides protection from myocardial infarction and ischemic stroke without affecting hemostasis (Krupka et al., 2016). The monoclonal antibody 3F7 targeting the FXIIa protease domain was shown to block artificial surfaceinduced thrombosis in mice and rabbits (Worm et al., 2015, Larsson et al., 2014).

The principal protease that activates FXII is plasma kallikrein (PKa). FXII has a major cleavage site between residues Arg353 and Val354, generating aFXIIa and additional cleavage sites are shown in Fig. 1. Auto-activation of FXII via contact with anionic surfaces 
in the presence of $\mathrm{Zn}^{2+}$ ions is a second route to forming $\alpha$ FXIIa (Ivanov et al., 2017). $\alpha$ FXIIa consists of two chains, the N-terminal heavy chain (353 residues, molecular weight (MW) $\sim 50 \mathrm{kDa}$ ) and the $\mathrm{C}$-terminal light chain (243 residues, MW $\sim 28 \mathrm{kDa}$ ) connected via a disulfide bridge between Cys340 and Cys467 (Fig. 1). A further cleavage of $\alpha$ FXIIa between residues Arg334-Asn335 produces $\beta$-factor XII ( $\beta$ FXIIa), which includes the light chain and nine residues of the heavy chain, termed the heavy chain remnant (HCR) (Pathak et al., 2015).

To characterise enzyme kinetics and perform protein crystallization experiments milligram quantities of purified $\beta$ FXIIa protease are required. The insect cell-based Drosophila expression system (DES, Invitrogen) is a preferred choice for heterologous protein expression due to high yields and eukaryotic post-translational modifications. Trypsin-like serine proteases have a high degree of flexibility in their loop structures, which is known to prevent crystallization. One approach to stabilize flexible proteins is described by Moon et al. 2010, utilising maltose binding protein (MBP) with surface entropy reduction (SER) mutations as an $\mathrm{N}$-terminal carrier protein to enhance protein crystallization (Moon et al., 2010). MBP has been reported to enhance the solubility of proteins when expressed as an N-terminal fusion in E. coli (Waugh, 2016, Jin et al., 2017) and mammalian expression systems (Reuten et al., 2016, Bokhove et al., 2016) but to date this has not been reported for insect cell expression systems. We describe the expression of the $\beta$ FXIIa protease domain as a secreted MBP fusion using DES, which facilitated the crystal structure determination for MBP- $\beta \mathrm{FXIIa}^{\mathrm{His}}$ in the active conformation in complex with peptidomimetic inhibitor D-PhePro-Arg chloromethylketone (PPACK). Another construct encoding recombinant $\beta \mathrm{FXIIa}^{\mathrm{His}}$ without the N-terminal fusion was also purified successfully and crystallized in the absence of inhibitor, stabilized by a crystal contact that mimics P1 (Arg) and P2 (Thr) residues from the activation loop of natural substrates FXII and PK. The latter $\beta$ FXIIa ${ }^{\text {His }}$ structure provides a rare example of a trapped protease-product complex and reveals a more open conformation of the S2 pocket than the MBP- $\beta$ FXIIa ${ }^{\text {His }}$-PPACK complex and the plasma purified $\beta$ FXIIa- 
benzamidine complex crystal structure (Fig. S1). We also utilised the $\beta$ FXIIa $^{\text {His }}$ crystal structure to examine the interactions formed with EcTI a kunitz type trypsin inhibitor and Infestin-4 a kazal-type inhibitor.

\section{Materials and methods}

All chemical and reagents were purchased from Sigma-Aldrich (Dorset, UK) unless otherwise stated and the purification columns used were purchased from GE Healthcare.

\subsection{Cloning and expression of $\beta$ FXIla ${ }^{H i s}$ and MBP- $\beta F X I l a^{H i s}$}

Based on the FXII mature protein sequence numbering, the gene fragment Asn335Ser596 coding for human $\beta$ FXIIa was cloned into a pMT-PURO (Addgene) expression plasmid alone or with a noncleavable N-terminal MBP tag (Moon et al., 2010, Ullah et al., 2008) for expression in the DES (Invitrogen, Thermo scientific Fischer, United States). To generate the MBP- $\beta$ FXIIa ${ }^{\text {His }}$ construct, $\beta$ FXIIa was fused to the MBP carrier protein with a five amino acid residues (AlaAlaAlaAlaSer) linker, as reported by Moon et.al., 2010 using the In-Fusion Advantage PCR Cloning KIT protocol (Clontech Laboratories, United States) and then subsequently cloned into the pMT-PURO vector. PCR primers were designed in order to share 15 bases of homology with the sequence at the ends of the linearized vector, MBP or FXII. The following set of primer pairs: 5'CTCGCTCGGGAGATCTAAAATCGAAGAAGGTAAACTGGA3' and 5'CGGGCCCTTAAGGCTAGCTGCGGCCGCATTAGTCTG3');

5'GCGGCCGCAGCTAGCGAATTCGGCCCGCTGAGCTGC3' and 5'ATGACCGGTACGCGTGCTCACGGTATGTTCGC3' were used for MBP and FXII, respectively. The PCR product encoding $\beta$ FXIIa ${ }^{\text {His }}$, was amplified using the primer pairs: 5'GTCGAGATCTCGGACCCCGCCTCAGTCC3';5'GCGC $A C G C G T$ GGAAACGGTGTGC TCCCGGA3'; then digested with the appropriate restriction enzyme (BgIII and MluI) and finally ligated into pMT-PURO between the BgIII and MluI restriction sites (Quick Ligation 
Kit from NEB). The pMT-PURO vector has an N-terminal Drosophila homologue of the immunoglobulin binding chaperone protein secretion signal (BiP) to enable secretion of recombinant proteins and a six His-tag sequence at the C-terminus (Iwaki \& Castellino, 2008). Plasmid DNA was purified with the commercially available Sigma-Aldrich Miniprep Kit and ethanol precipitated prior to transfection into D. melanogaster S2 cells. The cells were maintained in Schneider's Drosophila Medium (GIBCO, Fisher Scientific UK Ltd) supplemented with $10 \%$ fetal bovine serum (Sigma) and transfected $\left(2-3 \times 10^{6}\right.$ cells $/ \mathrm{mL}$, $>95 \%$ viability $5 \mathrm{~mL}$ in $25 \mathrm{~cm}^{2}$ flask (Nunc, Germany)) using standard calcium phosphate transfection methods. Selection of pMT-PURO plasmid transfected cells was performed using $10 \mu \mathrm{g} / \mathrm{ml}$ of puromycin antibiotic. Stable cells expressing the recombinant MBP- $\beta$ FXIIa ${ }^{\text {His }}$ were confirmed based on immunoreactivity with an anti-His tag monoclonal antibody using western blot (Towbin et al., 1979). When cells reached a density of $9 \times 10^{6}$ cells $/ \mathrm{mL}$ they were passaged by diluting 1:2 with Serum-free Express Five SFM insect culture medium (GIBCO, Fisher Scientific UK Ltd) supplemented with $20 \mathrm{mM}$ L-glutamine and finally cultured in a tissue culture shaker flask (Corning Incorporated, Corning, NY, USA) for large-scale expression with $\leq 2 \%$ serum. Protein expression was induced by supplementing culture media with $\mathrm{CuSO}_{4}$ to a final concentration of $0.5 \mathrm{mM}$. After 7 days at $28^{\circ} \mathrm{C}$, culture media were harvested, centrifuged and the supernatant was used for purification of the recombinant protein.

\subsection{Protein purification of $\beta F X I l a^{H i s}$ and MBP- $\beta F X I I a^{H i s}$}

Purification of recombinant $\beta \mathrm{FXIIa}^{\mathrm{His}}$ was initially performed by Ni-Sepharose affinity (His-Excel column) chromatography, with equilibration buffer containing $50 \mathrm{mM}$ HEPES, $\mathrm{pH} 8.3,500 \mathrm{mM} \mathrm{NaCl}, 5 \mathrm{mM}$ benzamidine and $10 \%$ glycerol, followed by a wash step with $20 \mathrm{mM}$ imidazole and eluted in presence of $500 \mathrm{mM}$ imidazole. The protein obtained from the Ni-affinity column was subject to anion exchange chromatography (Resource Q) with buffer A containing $20 \mathrm{mM}$ Tris $\mathrm{HCl}, \mathrm{pH} 8.3,5 \mathrm{mM}$ benzamidine and 
$10 \%$ glycerol, and buffer B containing buffer A with $1 \mathrm{M} \mathrm{NaCl}$. Gel filtration chromatography (HiLoad 16/60 Superdex 75 16/60 pg) was used for the final purification step with buffer containing $50 \mathrm{mM} \mathrm{Na-HEPES}$ at $\mathrm{pH} 8.3,500 \mathrm{mM} \mathrm{NaCl}, 5 \mathrm{mM}$ benzamidine, $10 \%$ glycerol. MBP- $\beta$ FXIIa $^{\text {His }}$ was initially purified using anion exchange chromatography with a Capto Q column with buffer A containing $20 \mathrm{mM}$ Tris $\mathrm{HCl}, \mathrm{pH} 8.0$ and buffer B containing $20 \mathrm{mM}$ Tris $\mathrm{HCl}, \mathrm{pH}$ 8.0, $1 \mathrm{M} \mathrm{NaCl}$. The second step comprised Ni-sepharose affinity chromatography with buffer A containing $50 \mathrm{mM}$ Tris $\mathrm{HCl}, \mathrm{pH} 8.0,200 \mathrm{mM} \mathrm{NaCl}$, $20 \mathrm{mM}$ imidazole and buffer B containing $50 \mathrm{mM}$ Tris $\mathrm{HCl}$ at $\mathrm{pH} 8.0,200 \mathrm{mM} \mathrm{NaCl}, 1 \mathrm{M}$ imidazole. This construct was finally purified by gel filtration (HiLoad 16/60 Superdex 200 16/60 pg) chromatography in buffer $20 \mathrm{mM}$ Tris HCl, pH 8.0, $200 \mathrm{mM} \mathrm{NaCl}, 40 \mathrm{mM}$ D-(+)maltose. The purified protein was concentrated in $20 \mathrm{mM}$ Tris $\mathrm{HCl} \mathrm{pH} 8.0,50 \mathrm{mM} \mathrm{NaCl}$ and $5 \mathrm{mM}$ D-(+)-maltose. The yield of MBP- $\beta \mathrm{FXIIa}^{\mathrm{His}}$ and $\beta \mathrm{FXIIa}{ }^{\mathrm{His}}$ varied between $1.3-2.0$ $\mathrm{mg} / \mathrm{L}$ and $0.8-1.0 \mathrm{mg} / \mathrm{L}$ of culture media, respectively.

\subsection{Assays of FXIla activity}

The enzymatic activity of $\beta$ FXIIa was assessed by measuring the change in absorbance at $405 \mathrm{~nm}$ associated with p-nitroaniline (pNA), formed upon cleavage of the chromogenic substrate, S-2302 (Chromogenix, Epsom, UK). 150 nM $\beta$ FXIIa was tested against increasing concentrations of S-2302 (0.4, 1, 2, 3, 4 and $6 \mathrm{mM})$. Commercial $\beta F X I I a$ (Enzyme Research Laboratories, Inc.), MBP- $\beta$ FXIIa $^{\text {His }}$ and $\beta \mathrm{FXIIa}^{\mathrm{His}}$ activity was assayed using $10 \mu \mathrm{L}$ of protein added to $10 \mu \mathrm{L}$ of substrate and diluted with $80 \mu \mathrm{L}$ of phosphatebuffered saline ( $\mathrm{pH} 7.4)$ at $37{ }^{\circ} \mathrm{C}$. The change in $\mathrm{OD}_{405}$ was monitored with a ENVISION multimode plate reader. An average of four independent experiments was used to determine the enzyme kinetics. Each substrate concentration was measured in quadruplicate (Fig. S2). The kinetic parameters were calculated from the experimental data using Graphpad Prism with the non-linear Michaelis-Menten algorithm. 


\section{4. $\beta F X I l a^{H i s}$ and MBP- $\beta F X I l a{ }^{H i s}$ crystallization and structure determination}

The purified $\beta$ FXIIa ${ }^{\text {His }}$ protein was concentrated to $14 \mathrm{mg} / \mathrm{mL}$ for crystallization trials using screens from Qiagen, Molecular Dimension and Hampton Research (Aliso Viejo, CA, USA) sitting drop plates. Crystals grew from conditions including $0.1 \mathrm{M}$ Tris- $\mathrm{HCl}, \mathrm{pH} 8.0$ and $1.5 \mathrm{M} \quad\left(\mathrm{NH}_{4}\right)_{2} \mathrm{SO}_{4}$ at $19{ }^{\circ} \mathrm{C}$. MBP- $\beta \mathrm{FXIIa}^{\mathrm{His}}$ was co-crystallized with the cholormethylketone based inhibitor, D-Phe-Pro-Arg-chloromethylketone (PPACK), which covalently modifies the Ser195 and His57 residues (Bode et al., 1989). The MBP-BFXII ${ }^{\mathrm{His}}$ PPACK complex was prepared by adding a 10-fold excess of PPACK and incubating the sample at $4{ }^{\circ} \mathrm{C}$ overnight. The crystallization of the MBP- $\beta$ FXIIa ${ }^{\text {His }}$-PPACK complex was carried out using two different protein concentrations, $8 \mathrm{mg} / \mathrm{mL}$ and $6 \mathrm{mg} / \mathrm{mL}$. Crystals of MBP- $\beta$ FXIIa ${ }^{\text {His }}$-PPACK suitable for data collection were grown in $0.1 \mathrm{M}$ sodium citrate, $\mathrm{pH}$ 5.6 with $10 \%$ PEG 4000 and $0.15 \mathrm{M} \mathrm{MgCl}_{2}$ at $19{ }^{\circ} \mathrm{C}$. Crystals obtained for both MBP$\beta F X I{ }^{\mathrm{His}}$-PPACK and $\beta$ FXIIa ${ }^{\mathrm{His}}$ were harvested, cryo-protected in the crystallization solution with $25 \%$ glycerol and flash cooled in liquid nitrogen. X-ray diffraction data were collected at the beamline I04 at Diamond Light Source, UK for both $\beta$ FXIIa ${ }^{\text {His }}$ and MBP- $\beta$ FXIIa ${ }^{\text {His }}$ PPACK, which diffracted to resolutions of $2.54 \AA$ and $4 \AA$, respectively. For MBP- $\beta F X I I a^{\text {His }}$ PPACK crystal, diffraction data were processed with XDS (Kabsch, 2010) then scaled and merged with AIMLESS (Evans \& Murshudov, 2013). The structure was determined by molecular replacement using PHASER (McCoy et al., 2007) from the CCP4 suite with search templates of the FXII protease domain crystal structure (PDB code: 4XDE) and the structure of MBP (PDB code: $1 \mathrm{HSJ}$ ). For $\beta$ FXIIa ${ }^{\text {His }}$ the diffraction data were processed with XIA2DIALS (Evans, 2006, Winter et al., 2018) and the structure was determined using molecular replacement with one molecule in the asymmetric unit. High quality electron density maps resulted and model building was performed with Coot (Emsley \& Cowtan, 2004) and refined with REFMAC (Murshudov et al., 2011) with crystallographic statistics presented in Table 1. 


\subsection{Interaction of $\beta F X I l a^{H i s}$ and EcTI}

\subsubsection{Gel filtration}

EcTI was purified as previously described (Batista et al., 1996). $\beta \mathrm{FXIIa}^{\mathrm{His}}$ was concentrated to $500 \mu \mathrm{M}$ and EcTI was added to a molar ratio of $1: 1$ then analyzed on analytical gel filtration column (Superdex 200 Increase 10/300 GL) in $20 \mathrm{mM}$ Tris $\mathrm{HCl}$, pH 8.0 and $200 \mathrm{mM} \mathrm{NaCl}, 5 \%$ glycerol. The EcTI- $\beta \mathrm{FXIIa}^{\mathrm{His}}$ complex peak fraction was analyzed by SDS-PAGE. Gel filtration standards (GE healthcare) were run to calibrate the column and calibration curve revealed a calculated molecular weight of the EcTI- $\beta$ FXIIa ${ }^{\text {His }}$ complex peak of $\sim 43 \mathrm{kDa}$.

\subsubsection{SPR binding studies}

SPR experiments were performed on a Biacore 3000 Instrument (BIAcore) at $25{ }^{\circ} \mathrm{C}$ as described previously (Wong et al., 2016). EcTI was covalently coupled on a CM5 sensor chip using an amine coupling kit (GE healthcare) with N-hydroxysuccinimide and N-ethyl-N(3-(dimethylamino)propyl) carbodiimide activation. EcTI was immobilized at approximately 300 response units (RU) and another flow cell was subjected to the identical immobilization procedure without EcTI protein for use as a control. The binding of $\beta$ FXII to EcTI was studied by injecting varying concentrations of $\beta$ FXII for 1200 seconds in a running buffer $(20$ mM HEPES buffer, $\mathrm{pH} 7.4,150 \mathrm{mM} \mathrm{NaCl}, 50 \mu \mathrm{M}$ EDTA, $0.005 \%$ polysorbate 20 ) at a flow rate of $50 \mu \mathrm{L} / \mathrm{min}$. To assess any nonspecific binding, the analyte $\beta$ FXIIa (both commercial $\beta$ FXIIa and recombinant $\beta$ FXIIa ${ }^{\text {His }}$ ) was also injected over the control flow cell. Binding curves were analyzed on the basis of the SPR RU recorded for each analyte protein concentration and data were processed with an appropriate fitting model using the BIA evaluation 4.1 to measure association and dissociation rate constants $\left(K_{\mathrm{D}}, k_{\mathrm{a}}\right.$ and $\left.k_{\mathrm{d}}\right)$. 


\subsubsection{Molecular docking}

Molecular docking was performed using CLUSPRO (Kozakov et al., 2017) as described previously (Hamad et al., 2017) and utilized the $\beta$ FXIIa ${ }^{\text {His }}$ crystal structure and the available crystal structure of EcTI (Protein Data Bank (PDB) code 4J2Y) and infestin-4 (PDB code 2ERW). Molecular graphics and figures were created using PyMOL.

\section{Results}

\subsection{Two recombinant forms of the active FXII protease}

Constructs for the MBP- $\beta$ FXIIa ${ }^{\text {His }}$ fusion and isolated protease domain $\beta \mathrm{FXIIa}^{\text {His }}$ (Fig. 1) were expressed in Schneider 2 (S2) cells using the pMT-PURO vector and subsequently purified from culture media using affinity, ion exchange and size exclusion chromatography. SDS-PAGE gels under reducing conditions revealed the purified $\beta \mathrm{FXIIa}^{\mathrm{His}}$ migrated as single band of $\sim 30 \mathrm{kDa}$ and MBP- $\beta$ FXIIa ${ }^{\mathrm{His}}$ as two bands of $\sim 30 \mathrm{kDa}$ and $\sim 40 \mathrm{kDa}$, corresponding to $\beta F X I I a$ and MBP, respectively, illustrating that during the purification the MBP- $\beta$ FXIIa ${ }^{\text {His }}$ activation loop has been cleaved.

To characterize the catalytic activity of MBP- $\beta$ FXIIa ${ }^{\mathrm{His}}$, the hydrolysis of chromogenic substrate S-2302 was monitored at $405 \mathrm{~nm}$. The kinetic parameters for recombinant MBP- $\beta$ FXIIa ${ }^{\text {His }}$, $\alpha$ FXIIa and $\beta$ FXIIa were calculated from the initial reaction rates as a function of the concentration of S-2302 and the Michaelis constant $\mathrm{K}_{\mathrm{m}}$ for MBP$\beta \mathrm{FXIIa}^{\mathrm{His}}$ is comparable to commercial plasma purified $\beta$ FXIIa and $\alpha$ FXIIa (Fig. S2; Table 1). 


\subsection{Crystal structure of $\beta F X I l a^{\text {His }}$}

$\beta$ FXIIa $^{\text {His }}$ crystallized in the tetrahedral space group $\mathrm{P}_{1}{ }_{1} 22$ and data were collected to $2.54 \AA$ resolution with one molecule in the asymmetric unit. The crystal structure of $\beta$ FXIIa ${ }^{\text {His }}$ spans residues $\mathrm{Asn}^{335}{ }^{-\mathrm{Ser}^{596}}$ (native FXII sequence numbering) with high-quality electron density and crystallographic refinement statistics (Table 2, Fig. S3). Our cloning strategy using the MluI restriction site resulted in the recombinant $\beta \mathrm{FXIIa}{ }^{\text {His }}$ having four extra residues at the $\mathrm{C}$ terminus prior to the His-tag with sequence $\mathrm{Thr}^{1}-\mathrm{Arg}^{2}-\mathrm{Thr}^{3}-\mathrm{Gly}^{4}-(6 \mathrm{X}) \mathrm{His}$ (Fig. 1). We have followed a chymotrypsin-based numbering system throughout for FXII residues unless otherwise indicated and the $\mathrm{C}$-terminal non-native amino acids are numbered in superscript (i.e. $\mathrm{Thr}^{1}$ ) prior to the His-tag. Unexpectedly, $\mathrm{Thr}^{1}-\mathrm{Arg}^{2}$ was observed in the electron density maps forming a crystal contact, whereas the remaining C-terminal residues $\mathrm{Thr}^{3}$-Gly ${ }^{4}$ (6X)His were absent (Fig. 2A, S3). The $\mathrm{Arg}^{2}$ side chain is observed in the $\mathrm{S} 1$ binding pocket and its guanidinium group forms the characteristic salt bridge to the Asp189 carboxylate (Fig. $2 B$ and $\mathrm{S} 3$ ). The $\mathrm{Thr}^{1}$ side chain projects into the S2 pocket, formed by side chains of His57, Tyr99, Phe94, Pro96 and Gln60 and the Thr ${ }^{1}$ main chain carbonyl forms a hydrogen bond to the side chain of Gln192 (Fig. 2B). FXII residue Gly216 from the S2 pocket also forms two hydrogen bonds to the main chain of Ser244 from the crystal contact which are characteristic S2 pocket interactions for bound peptide mimetic P3 residues observed in other protease complex crystal structures (Kristensen et al., 2016, Bode et al., 1989). Thus the BXXIIa $^{\text {His }}$ structure likely represents an autoproteolytic cleavage of the $\mathrm{Arg}^{2}-\mathrm{Thr}^{3}$ peptide bond to produce a crystal contact that presents a product complex with the C-terminal $\mathrm{Arg}^{2}$ carboxylate forming electrostatic interactions with the $\beta$ FXIIa $^{\text {His }}$ oxyanion hole. A superposition of the recombinant $\beta$ FXIIa ${ }^{\text {His }}$ structure with the plasma purified $\beta$ FXIIabenzamidine complex structure (Dementiev et al., 2018) illustrates key differences arise in the area of the substrate selectivity S2 pocket (Fig. S1) as outlined below. 


\subsection{Comparison of $\beta F X I l a{ }^{H i s}$ with FXII zymogen protease structures}

We have previously reported two zymogen-like crystal structures for the FXII protease spanning the protease domain alone (amino acids 354 to 596) (Pathak et al., 2015). These are termed (i) FXIIc (PDB code: 4XDE), which corresponds to a construct where the N-terminus is blocked by addition of two extra amino acids Arg-Ser and has very low enzymatic activity and (ii) FXIIac, (PDB code: 4XE4) which has the native N-terminal Val354 residue and 10fold higher enzyme activity compared to FXIIc but 1000-fold lower activity compared to $\beta$ FXIIa. FXIIac is lacking the additional N-terminal residues of the HCR found in $\beta$ FXIIa, which we speculated are required for efficient catalytic activity (Pathak et al., 2015). Comparison of FXIIa $^{\mathrm{His}}$ with FXIIc reveals the expected major differences in the conformation of the 140-loop (also termed the autolysis loop), 180-loop and 220-loop as is typical for trypsin-like serine proteases (Fig. 2C). In $\beta \mathrm{FXIIa}^{\mathrm{His}}$, the 140-loop forms interactions with the C-terminal $\beta$-barrel, which is in contrast to the 140 -loop that interacts with the N-terminal $\beta$-barrel in both the FXIIc and FXIIac structures.

Additional changes not previously observed are the movement of the 99-loop away from the catalytic serine in $\beta$ FXIIa ${ }^{\text {His }}$ and the Tyr99 sidechain has an alternate conformation compared to the FXIIc zymogen protease structure. The consequence is the Tyr99 sidechain moves away from the close packing interaction with His57 and thus opens up the S2 pocket in $\beta$ FXIIa (Fig. 2D,E), which notably comes at the expense of the S3 pocket as the Tyr99 sidechain partially occludes this by packing against Trp215.

\section{4 $\beta F X I l a$ surface charge features}

We have previously observed ridges of negative charge (termed R1 and R2) in the FXIIc structure surrounding the catalytic residues (Pathak et al., 2015). A comparison of FXIIc with the active $\beta \mathrm{FXIIa}^{\mathrm{His}}$ structure reveals a substantial re-arrangement of charge at the R2 ridge 140-loop residues Glu151B, Glu151E and Glu151F, which move from the C-terminal $\beta$ - 
barrel closer to the S1 pocket and contributes to the negative charge flanking the region of the S1' pocket together with residue Asp60A (Fig. $3 A$ and $B$ ). The 140-loop is known to contribute to the interaction of proteases with serpins (Gong et al., 2015, Baglin et al., 2002) and thus the differences in this region observed between FXIIa and proteases such as FXIa and thrombin from the extrinsic pathway may contribute to the selectivity of inhibitor binding which is examined below.

Another distinctive feature observed in the zymogen-like FXIIc structure is the hydrophobic $\mathrm{H} 1$ pocket, which is positioned at the centre of the $\mathrm{N}$-terminal $\beta$-barrel and opposite to the $\mathrm{S} 1$ pocket. In the $\beta \mathrm{FXIIa}{ }^{\mathrm{His}}$ structure the $\mathrm{H} 1$ pocket is closed due to the position of Trp35, which forms a lid on the pocket and this is likely to be the dominant conformation (Fig. 3A).

\subsection{MBP-ßBFXIla ${ }^{\mathrm{His}}$-PPACK crystal structure}

We next sought to determine the structure of a peptide mimetic complex and utilised the commercially available inhibitors PCK (Pro-Phe-Arg) and PPACK (Phe-Pro-Arg). These did not co-crystallize with $\beta$ FXIIa ${ }^{\text {His }}$ so we engineered the MBP fusion construct to facilitate structure determination with inhibitors. MBP- $\beta$ FXIIa ${ }^{\text {His }}$ crystals were obtained through cocrystallization only with the PPACK inhibitor and the structure was determined by molecular replacement. Two copies of the MBP- $\beta$ FXIIa ${ }^{\text {His }}$ fusion were placed in the resulting highquality electron density map and the refined model (Table 2) showed PPACK bound to the active site, and the FXII Asn74 N-linked glycan residues are also modelled (Fig. S3). Fig. $4 A$ shows the overall structure of the MBP- $\beta$ FXIIa ${ }^{\text {His }}$ fusion with the $\beta$ FXIIa ${ }^{\text {His }}$ protease domain (coloured as a rainbow) positioned on top of the MBP (colored in green). The amino acid linker of sequence AlaAlaAlaAlaSer connecting the C-terminus of MBP to the N-terminus of $\beta \mathrm{FXIIa}^{\mathrm{His}}$ is partially observed in the electron density (Fig. $4 A, \mathrm{~S} 3$ ) and is assumed to be 
flexible (shown as a black dotted line). The 5 amino acids from the HCR fragment including the Cys340-Cys467 disulfide are also observed (Fig. 4A).

Unexpectedly multiple interactions are formed between $\mathrm{MBP}$ and $\beta \mathrm{FXIIa}^{\mathrm{His}}$ extending over a surface area of $654.8 \AA^{2}$, (calculated using PISA) (Fig. 4B). For both copies of MBP- $\beta$ FXIIa $^{\text {His }}$ the same organisation of MBP and $\beta$ FXIIa $^{\text {His }}$ is observed and is superposable with an r.m.s.d. of $0.688 \AA$ (for $614 \mathrm{C}-\alpha$ atoms). This MBP- $\beta$ FXIIa ${ }^{\text {His }}$ interface occurs between three irregular loops from the FXII protease domain $\beta$-barrels and helices $\alpha 2$, $\alpha 12$ of MBP. From MBP helix $\alpha 2$ Lys46 forms a salt bridge to Asp111 from FXII, MBP Lys42 forms hydrogen bonds to the main chain carbonyl groups of residues Glu78 and Cys 80 and MBP Gln49 (helix $\alpha 2$ ) forms a hydrogen bond to the carbonyl of FXII Leu114 (Fig. 4B). A second series of interactions originates from MBP helix $\alpha 12$ with a salt bridge between MBP Arg354 and FXII Glu71 and hydrogen bonding interactions formed between the side chain groups of MBP Asn349 and Thr345 with the main chain nitrogen of FXII Arg24 and the side chain of Tyr117, respectively. The MBP Tyr341 side chain forms a hydrophobic interaction with FXII Pro116, MBP Asp207 forms a salt bridge to FXII Arg72 and the MBP Asn150 side chain hydrogen bonds to the FXII Glu78 side chain. These interactions result in the FXII protease covering the entrance to the MBP pocket where maltose binds and may explain why this additive was required for the purification and crystallization experiments.

In the MBP- $\beta F X I I a^{H i s}$-PPACK structure, the Arg-Pro residues form characteristic interactions with the FXIIa S1 and S2 pockets as observed in the thrombin complex crystal structure, however, the P3 PPACK Phe residue projects away from the protease and is involved in a crystal contact. This differs from the thrombin-PPACK complex where the Phe residue is inserted in the $\mathrm{S} 3$ pocket (Fig. 4C) (Bode et al., 1989). 


\subsection{The complex of $\beta F X I l a{ }^{H i s}$ with inhibitor EcTI}

We next chose to characterise whether the recombinant insect cell expressed $\beta \mathrm{FXIIa}^{\mathrm{His}}$ would interact with a kunitz type protease inhibitor and perform a comparison with the plasma purified $\beta$ FXIIa, which will have a larger glycan structure. EcTI is a trypsin inhibitor derived from the plant Enterolobium contortisiliquum, which has been reported as inhibiting FXIIa activity and a crystal structure of EcTI in complex with trypsin is available (Zhou et al., 2013). To characterise the $\beta$ FXIIa-EcTI complex gel filtration (Superdex 200 Increase) chromatography was performed with an equimolar mixture of $\beta \mathrm{FXIIa}^{\mathrm{His}}$ and EcTI, with the elution profile shown in Fig. $5 A$. This revealed a $\beta$ FXIIa $^{\text {His }}$-EcTI complex of approximately $43 \mathrm{kDa}$ with an excess of EcTI identified as a separate peak ( 20 kDa) confirmed by SDS-PAGE of the resulting fractions (Fig. $5 A$ ).

Next, surface plasmon resonance (SPR) was performed to quantify the $\beta \mathrm{FXIIa}^{\mathrm{His}}$ EcTI binding. As shown in Fig. 5B, SPR binding curves demonstrated concentrationdependent binding. The data were fitted using the 1:1 binding model with mass transfer to derive values of $\mathrm{K}_{D}, k_{\mathrm{a}}$ and $k_{\mathrm{d}}$. The $\mathrm{K}_{D}, \mathrm{k}_{a}$ and $\mathrm{k}_{d}$ for EcTI binding to the plasma purified $\beta$ FXII ${ }^{\mathrm{His}}$ were $2.68 \times 10^{-7} \mathrm{M}, 1.02 \times 10^{4} \mathrm{M}^{-1} \mathrm{~s}^{-1}$, and $2.71 \times 10^{-3} \mathrm{~s}^{-1}$, respectively. These values are comparable to EcTI binding to recombinant $\beta$ FXII ${ }^{\text {His }}$ and the $\mathrm{K}_{D}, \mathrm{k}_{a}$ and $\mathrm{k}_{d}$ values were $3.38 \times 10^{-7} \mathrm{M}, 8.17 \times 10^{3}, \mathrm{M}^{-1} \mathrm{~s}^{-1}$, and $2.76 \times 10^{-3} \mathrm{~s}^{-1}$, respectively.

To understand the molecular basis of the Kunitz-type inhibitor complex with $\beta$ FXIIa we performed molecular docking with the crystal structures of $\beta$ FXIIa ${ }^{\mathrm{His}}$ and EcTI (pdb code: 4J2Y) using the program Cluspro (Kozakov et al., 2017). Inspection of the top ranked docking solution identified a canonical complex whereby the EcTI residue Arg64 was inserted into the S1 pocket of $\beta$ FXIIa ${ }^{\text {His }}$ with scores of -769.4 and -962.1 , representing centre and lowest energy scores, respectively, for 274 members in the largest cluster size (the second ranked solution had 101 members), and no steric clashes were observed. 
Fig. $6 A, B$ shows a cartoon diagram of the predicted $\beta$ FXIIa $^{\text {His }}$-EcTI complex and Fig. $6 C$ a surface charge distribution of $\beta$ FXIIa ${ }^{\text {His }}$ with EcTI as a cartoon diagram. Table S1 lists the key electrostatic and hydrogen bond interactions at the interface, burying a total surface area of $1207 \AA^{2}$ (pdbe-PISA) in comparison to $988 \AA^{2}$ observed in the trypsin-EcTI complex (pdb code: 4J2K). The EcTI Arg64 side-chain extends into the S1 pocket of the FXIIa catalytic domain forming a salt bridge with the S1 pocket residue Asp189 side-chain carboxyl (chymotrypsin numbering used) (Fig. 6B). Additional interactions flanking the P1 Arg64 include Pro63 interacting with the S2 pocket (Fig. 6D) which is similar to interactions observed in the MBP- $\beta$ FXIIa ${ }^{\text {His }}$-PPACK complex.

\subsection{P2 substrate preference of $\beta F X I I a$}

FXIIa cleaves three substrates FXII, PK, FXI and substrate selection is driven by a combination of exosite and subsite interactions that are poorly understood. An alignment of the sequences in the area of the cleavage site is shown in Table 3. All have Arg at the P1 position, but at the P2 position there is greater variability as FXI has Pro and FXII, PK both have a Thr residue. High throughput protease specificity profiling using a diverse short peptide based fluorogenic substrate microarray confirmed the requirement of Arg at P1 and revealed a FXIIa preference for Phe and Thr at the P2 position, which is consistent with the sequence Pro-Phe-Arg of the widely used FXIIa substrate S-2302 (Gosalia et al., 2006, Zhou et al., 2013). Table 3 shows FXII inhibitors follow this preference for Phe and Thr at the P2 position (PCK, infestin-4, and bicyclic peptide (Middendorp et al., 2017)) and also mimic its substrate FXI with Pro in the P2 (CTI and EcTI). This more open S2 pocket observed in both MBP- $\beta$ FXIIa ${ }^{\text {His }}$ and $\beta$ FXIIa $^{\text {His }}$ presents a framework that explains the FXIIa substrate preference for a bulky Phe sidechain at the $\mathrm{P} 2$ position. Why the natural substrates utilise Thr at P2 more commonly and do not utilise Phe at P2 is unclear, but as discussed below, it is likely that exogenous inhibitor infestin-4 does utilise the open S2 pocket to interact with a P2 Phe sidechain. 


\subsection{Docking of $\beta F X I l a{ }^{H i s}$ with Infestin-4}

The exogenous inhibitor Infestin-4 is derived from the blood sucking kissing bug and has been characterised in detail as a FXIIa inhibitor which has also been optimised for selectivity. Previous studies have performed docking on Infestin-4 using a model of FXIIa and presented a complex which places the Infestin-4 P2 Phe9 sidechain in the S1' pocket and the P1' Asn11 in the S2 pocket (Kolyadko et al., 2015). A crystal structure of Infestin-4 was utilised in docking simulations with the $\beta$ FXIIa ${ }^{\text {His }}$ crystal structure. Figure 7 shows the highest scoring complex with the Infestin-4 P1 Arg and P2 Phe inserted into the $\beta$ FXIIa ${ }^{\text {His }}$ S1 and S2 pocket respectively. Interestingly three highly complementary salt bridges are formed between infestin-4 residues Lys36, Lys21 and Arg40 with $\beta$ FXIIa ${ }^{\text {His }}$ residues Asp222 from 220-loop and E151B, E151E from the 140-loop, respectively. This orientation is the reverse of the Kolyadko et al., 2015 Infestin-4:FXIIa docked complex and highlights the importance of consdering the $\beta \mathrm{FXIIa}^{\mathrm{His}}$ crystal structure with an open S2 pocket as a template for docking studies.

\section{Discussion}

Efforts are currently underway to design selective inhibitors for FXIIa as novel treatments for thrombotic and inflammatory disorders (Middendorp et al., 2017, Kolyadko et al., 2015, Krupka et al., 2016, Campos et al., 2012). To investigate the FXIIa protease structure in complex with peptidomimetic inhibitors we developed vectors and an insect cell based protocol for recombinant expression and purification of the $\beta$ FXIIa protease domain achieving yields suitable for enzyme kinetic and protein crystallography experiments. MBP$\beta$ FXIIa $^{\text {His }}$ crystals were obtained through co-crystallization experiments with the PPACK inhibitor. PPACK is a selective inhibitor of thrombin and a comparison of the PPACK-MBP$\beta$ FXIIa $^{\text {His }}$ structure with the reported PPACK-thrombin complex (Bode et al., 1989) revealed 
the PPACK P3 Phe residue forms interactions with residues in the S3 pocket in the thrombin complex, whereas in the MBP- $\beta$ FXIIa ${ }^{\text {His }}$ complex the Phe residue is projecting outwards and not forming significant interactions with contacts exclusively through the PPACK Arg and Pro residues which is reminiscent of the FIXa-PPACK complex (Kristensen et al., 2016).

A second construct $\beta \mathrm{FXIIa}^{\mathrm{His}}$ crystallized in the absence of an inhibitor, but here the active conformation was stabilized by Thr-Arg residues from an adjacent molecule in the crystal. This Thr-Arg sequence mimics the natural FXIIa substrate P1-P2 cleavage site residues present in prekallikrein and FXII activation loop sequences. Previous studies utilizing tripeptide substrates have determined the FXIIa optimal residues at P2 to be Phe or Thr, and the substrate S-2302 widely used to monitor FXIIa activity has the sequence ProPhe-Arg. The MBP- $\beta$ FXIIa ${ }^{\text {His }}$-PPACK (Pro-Arg) and $\beta$ FXIIa ${ }^{\text {His }}$-Thr-Arg substrate mimetic structures reveal that the Tyr99 residue alters its position from packing against His57 (observed in the zymogen FXIIc structure), which effectively opens up the S2 pocket. This movement of Tyr99 comes at the expense of the S3 pocket, which is partially occluded by packing of Tyr99 against Trp215.

Infestin-4 and a bicyclic peptide are selective protein/peptide based inhibitors of FXIIa which both utilise Phe residue at the P2 position (Middendorp et al., 2017, Kolyadko et al., 2015, Krupka et al., 2016, Campos et al., 2012). The more open S2 pocket provides a model for the preference of the bulky Phe residue at P2 and future studies involving a crystal structure with bound substrate mimetic or exogenous inhibitor will be required to demonstrate how this pocket is utilised. A series of crystal structures have been reported for protease domains that have a similar S2 pocket and residue Tyr99 (FXa, tPa and FIXa), however, most of these have the closed configuration of S2 with Tyr99 packing against His57, implying this transiently opens only in the presence of a substrate mimetic. A comparison of the recombinant $\beta$ FXIIa $^{\text {His }}$-Thr-Arg structure with the benzamidine- $\beta$ FXIIa structure reveals the latter also has a closed S2 pocket (Dementiev et al., 2018). 
The role of the distinctive negatively charged ridges in the 140 and 220-loops observed surrounding the FXII active site is unknown(Pathak et al., 2015), but the substrates FXI (Mohammed et al., 2018), PK (Hooley et al., 2007) and the natural inhibitor serpin C1 inhibitor have positively charged residues (Huntington, 2011, Beinrohr et al., 2007, Hamad et al., 2017) that may provide electrostatic interactions with $\beta$ FXIIa. Overall, these $\beta$ FXIIa structures provide a framework to understand substrate selectivity and for rational design of inhibitors to target thrombotic and inflammatory diseases.

\section{Author contributions}

R Manna determined the MBP- $\beta$ FXIIa ${ }^{\text {His }}$ structure and M Pathak determined the $\beta \mathrm{FXIIa}^{\mathrm{His}}$ structure and contributed to preparation of the draft manuscript. D Belviso and $\mathrm{C} \mathrm{Li}$ performed crystallographic refinement analysis. R Bonturi and Maria Luiza V Oliva isolated and purified EcTI. B Hamad performed enzyme kinetic data analysis. J Emsley, LV Dekker, PM Fischer, I Dreveny conceived and designed the study, generated, analyzed and collected/interpreted data, wrote and revised the intellectual content and generated the final manuscript version.

\section{Acknowledgements}

Crystallographic data were obtained with the support of Diamond Light Source using the beamlines I04 and I24.

PDB reference The atomic coordinates and structure factors of $\beta$ FXIIa ${ }^{\mathrm{His}}$, (pdb code: $6 \mathrm{GT} 6$ ) and MBP-BFXIIa ${ }^{\text {His }}$ (pdb code: 6QF7) have been deposited in the Protein Data Bank (http://www.rcsb.org).

\section{Funding information}


This work was supported by the British Heart Foundation studentship award FS/11/61/28941 to Rosa Manna and program grant programme Grant no. RG/12/9/29775 to JE. Also grant Fundação de Amparo à Pesquisa do Estado de São (FAPESP) [2017/07972-9 and 2017/06630-7]; Coordenação de Aperfeiçoamento de Pessoal de Nível Superior (CAPES) [23038.007762/2014-32, AUXPE 140/2015] to MLVO. The authors declare that they have no conflicts of interest with the contents of this article.

Abbreviations. FXII, Factor XII, PK, Prekallikrein; PDB, Protein Data Bank, CTI, Corn Trypsin inhibitor, MBP, Maltose binding protein 


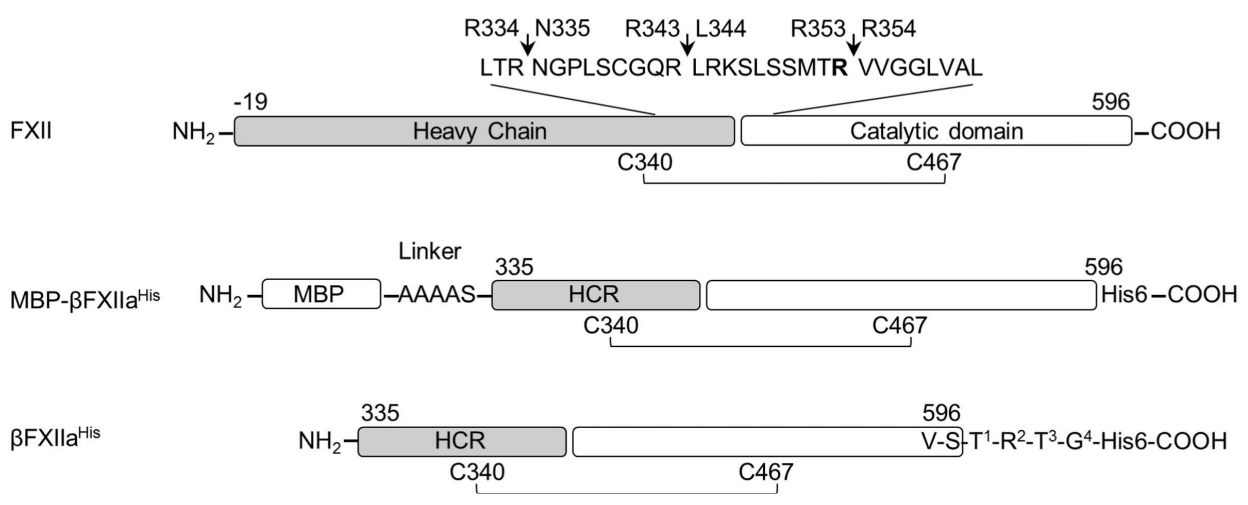

Figure 1. FXII domain organization and protein expression construct designs. Schematic representation of the FXII domain organization (top) with the disulfide bridge (Cys340Cys467) between heavy chain and catalytic domain is shown. The principal activating cleavage site is depicted as an arrow between residues Arg353-Val354. Other cleavage sites are depicted with Arg334-Asn335 resulting in the formation of $\beta$ FXIIa. Below this is the MBP- $\beta$ FXIIa ${ }^{\mathrm{His}}$ and $\beta$ FXIIa ${ }^{\mathrm{His}}$ constructs used to generate recombinant $\beta$ FXIIa. HCR denotes the 9 amino acid heavy chain remnant found in $\beta$ FXIIa. 
(a)

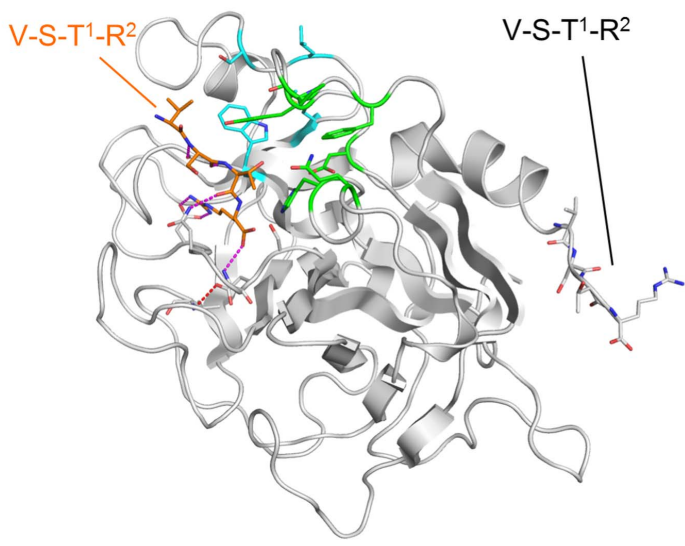

(b)

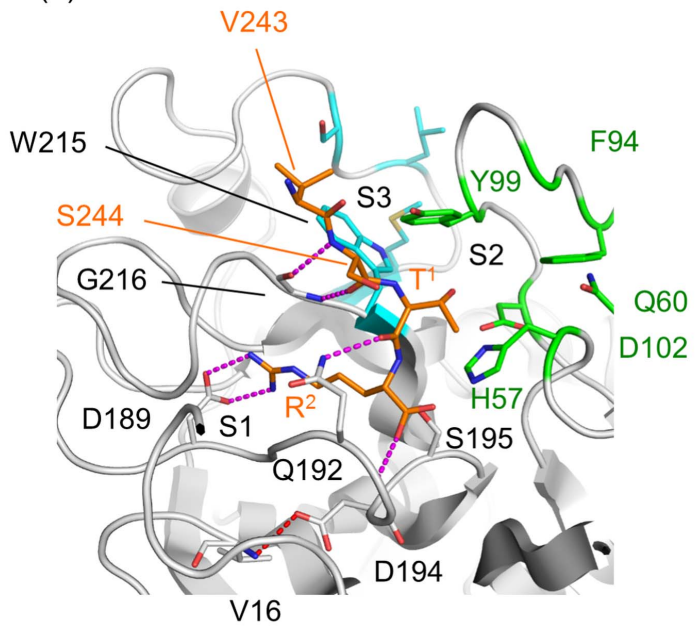

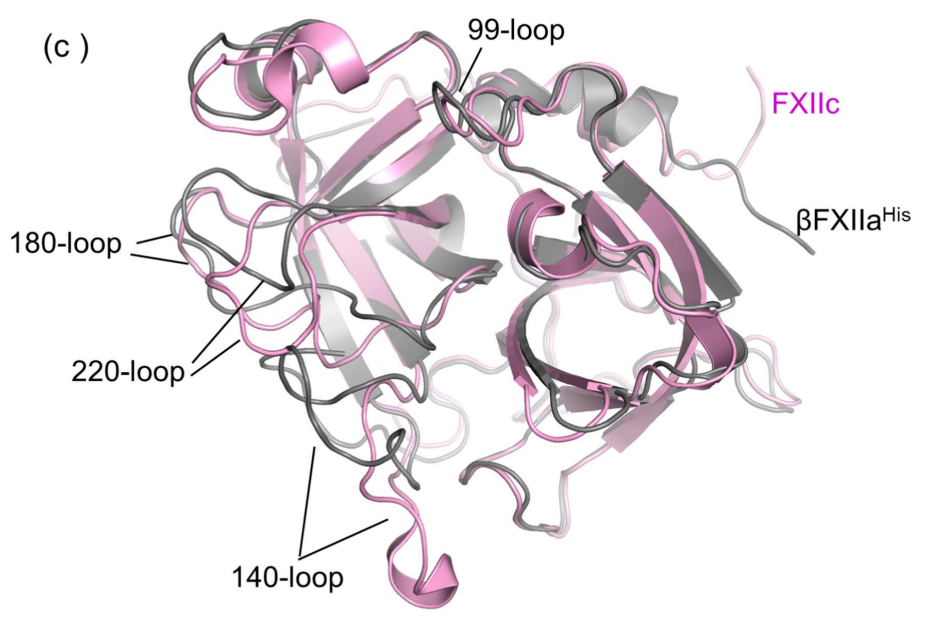

(d)

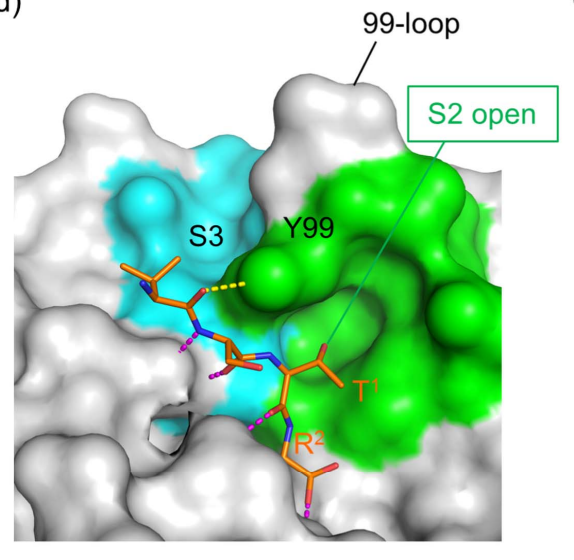

(e)

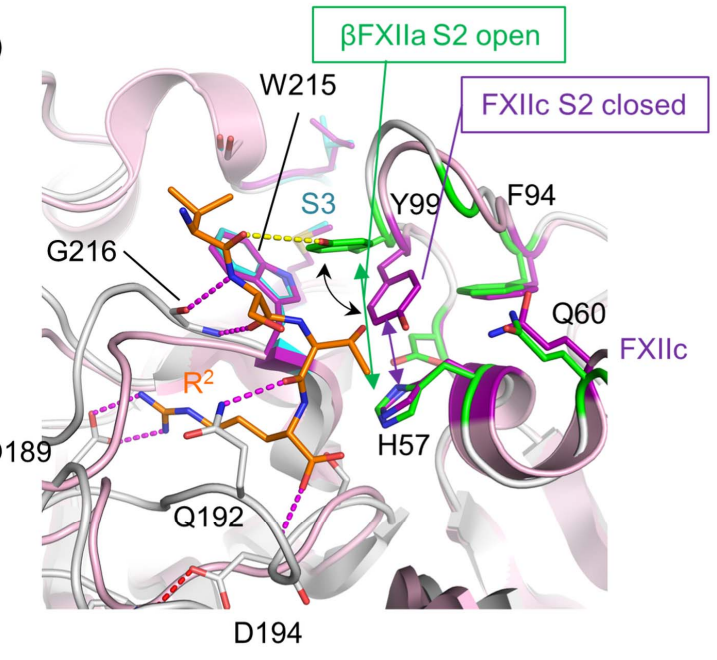


Figure 2. Structure of $\beta$ FXIIa ${ }^{\text {His }}$. (a) Cartoon diagram of the $\beta \mathrm{FXIIa}^{\mathrm{His}}$ active site is shown occupied by four amino acids (sticks colored orange) from the C-terminus of an adjacent molecule in the crystal. $\mathrm{Thr}^{1}-\mathrm{Arg}^{2}$ are numbered thus as they are not derived from the FXII sequence but from the $\mathrm{C}$-terminal regions of the expression construct. Residues from the $\mathrm{S} 2$ pocket are shown as sticks in green and S3 in cyan. (b) A close up of the structure in $A$ shown with electrostatic interactions between V-S-T-R residues and residues from the $\beta$ FXIIa ${ }^{\text {His }} \mathrm{S} 1$, $\mathrm{S} 2$ and S3 pockets illustrated as purple dotted lines. (c) Cartoon diagram of $\beta$ FXIIa ${ }^{\mathrm{His}}$ (grey) superposed onto the FXIIc structure (purple) with key loops altering conformation labeled (140-loop represents residues in the range of residue 140 using chymotrypsin numbering which is also known as the autolysis loop). (d) Surface representation of the S2 and S3 pockets in a similar view as shown in $B$. Close contact of the Tyr99 hydroxyl with the V-S-T$\mathrm{R}$ main chain carbonyl is shown as a yellow dotted line. (e) The S2 pocket in $\beta \mathrm{FXIIa}^{\mathrm{His}}$ with V-S-T-R residues (orange sticks) is shown superposed onto the FXIIc structure (purple) illustrating the positional change of residue Tyr99 annotated as an open or closed S2 pocket. 
(a)

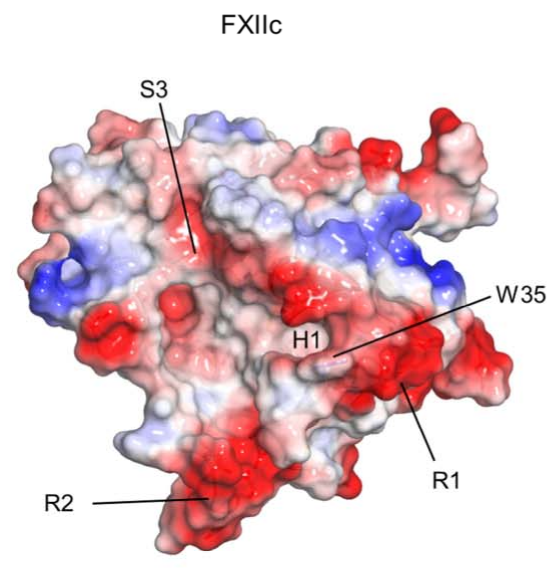

$-63.775-63.775$

(b)

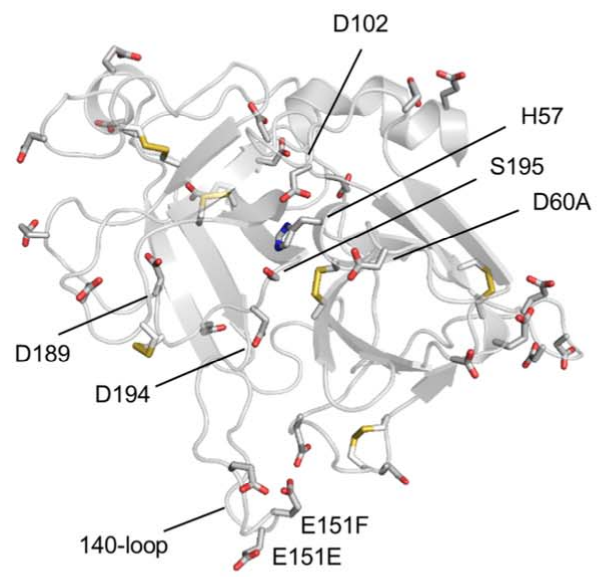

$\beta$ FXIla ${ }^{\mathrm{His}}$
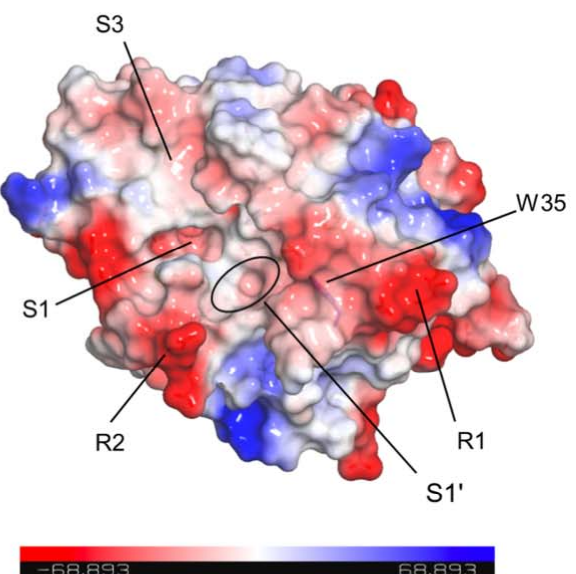

$\beta F X I l a H i s$

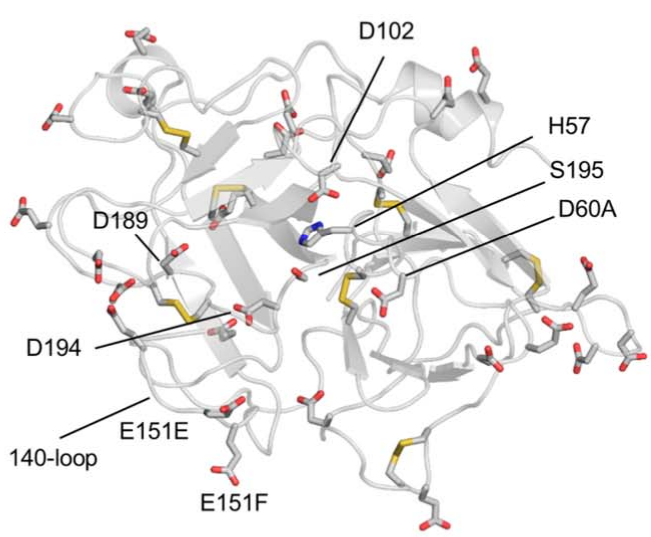

Figure 3. Changes in the overall surface charge distribution in the $\beta \mathrm{FXIIa}^{\mathrm{His}}$ structure compared to the zymogen like FXIIc structure. (a) Electrostatic (red=negative; blue= positive) charge distribution in both $\beta$ FXIIa (right) and FXIIc (left) with a large movement of the ridge of negative charge labeled R2 is shown. (b) Cartoon diagram showing the distribution of acidic residues highlighted as sticks in both the zymogen FXIIc (left) and the active $\beta$ FXIIa (right). 
(a)
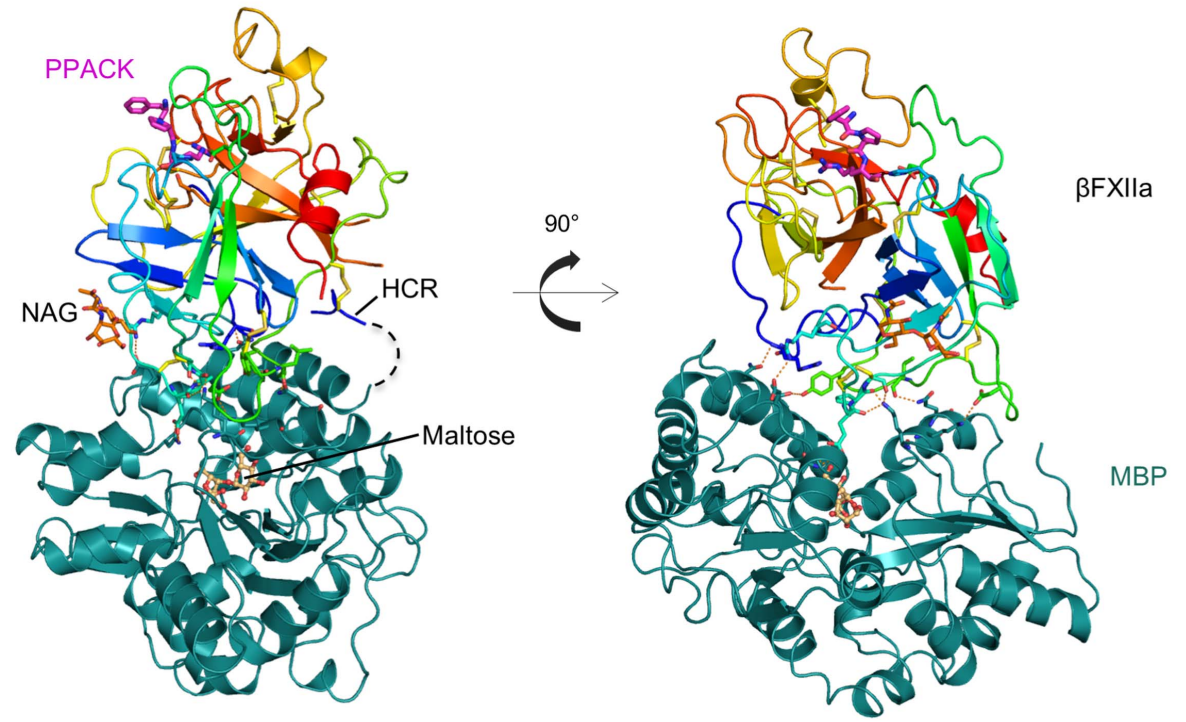

(b)

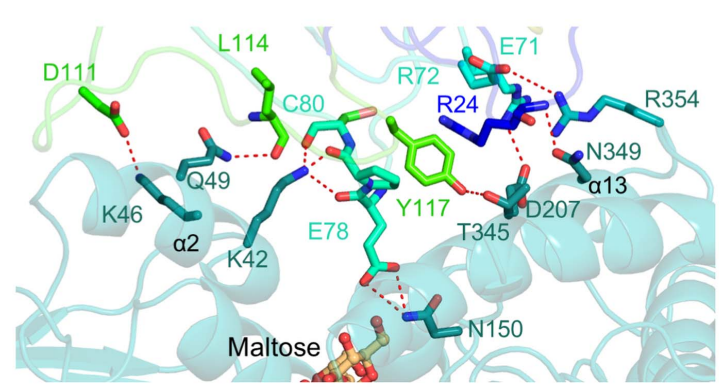

(c)

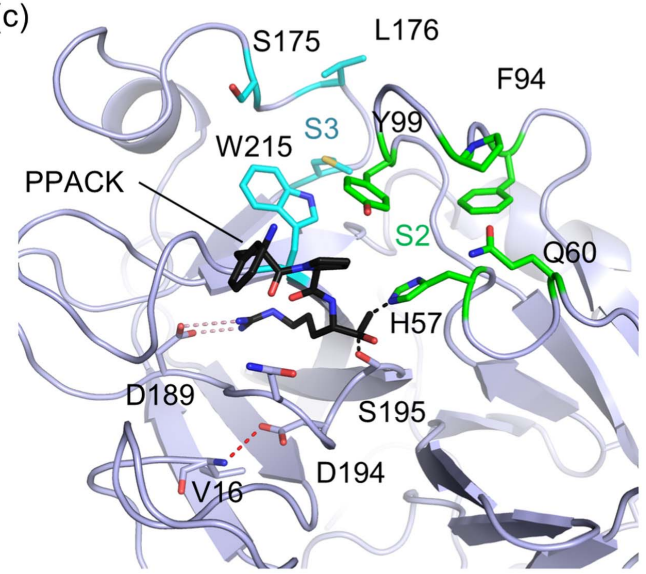

(d)

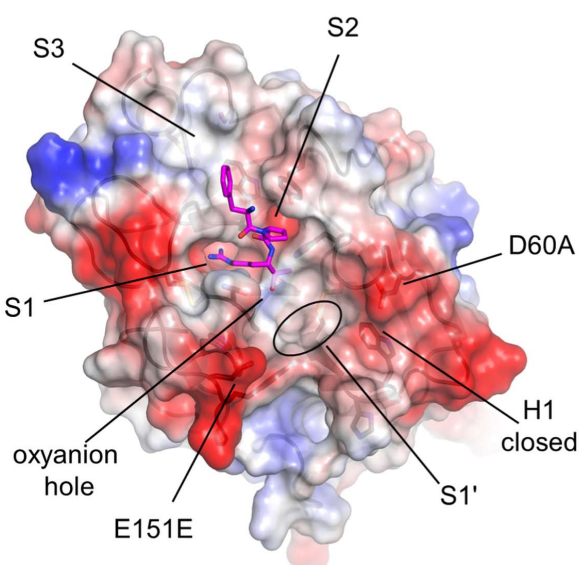


Figure 4. Structure of the MBP- $\beta$ FXIIa ${ }^{\text {His }}$ crystal structure complexed with PPACK. (a) Cartoon representation of the crystal structure of the MBP- $\beta$ FXIIa ${ }^{\text {His }}$-PPACK complex. MBP shown in dark green and $\beta$ FXIIa ${ }^{\text {His }}$ in rainbow with HCR indicated and N-linked glycan residues as sticks in orange and PPACK (purple). The maltose bound to MBP is drawn in ball and stick in yellow. (b) Ribbon diagram of the interactions occurring at the interface between $\mathrm{MBP}$ and the $\beta \mathrm{FXIIa}{ }^{\mathrm{His}}$ fusion partner. The interacting residues are drawn in sticks with electrostatic interactions depicted as dashed lines. (c) Detailed interactions of PPACK (black) with key residues shown as sticks forming interactions with the S1 pocket (dashed lines). The dotted circle shows the oxyanion hole and Asp189 residue forming the base of the S1 pocket is also shown. (d) MBP- $\beta$ FXIIa ${ }^{\text {His }}$ protease surface charged representation (negative $=$ red, positive=blue). The PPACK P1 arginine is bound in the S1 pocket (purple sticks). 
(a)

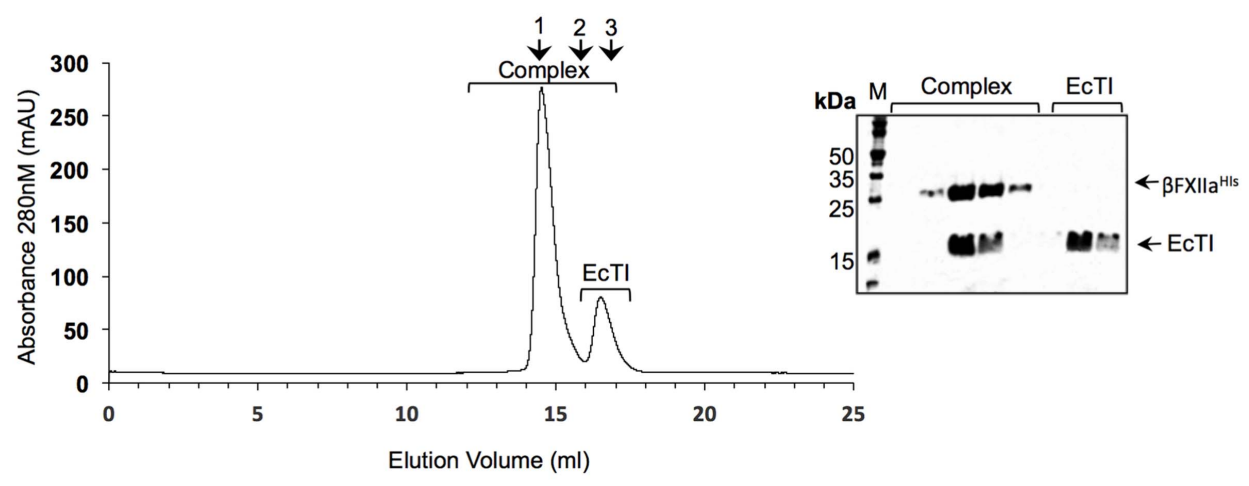

(b)

ßFXIla-EcTI

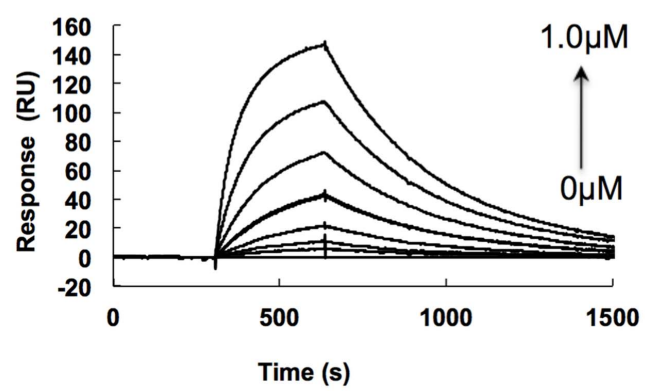

$\beta$ FXIIaHis-EcTI

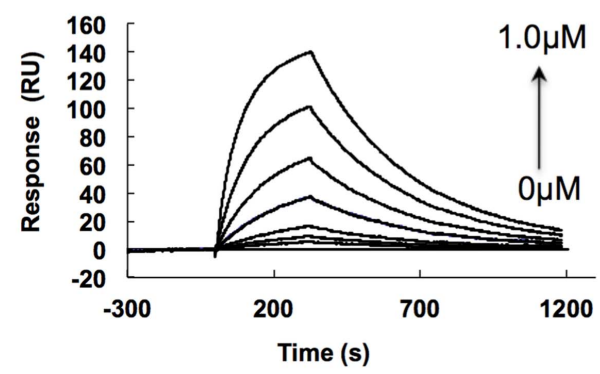

Figure 5. $\beta$ FXIIa binding to kunitz type inhibitor EcTI. (a) The $\beta$ FXIIa ${ }^{\text {His }}$-EcTI complex was analyzed by gel filtration. Arrows numbered 1, 2, and 3 indicate the elution volume of molecular weight standards of $43 \mathrm{kDa}, 22 \mathrm{kDa}$ and $13.7 \mathrm{kDa}$ respectively. The panel on the left shows the coomassie stained SDS-PAGE gel under reducing conditions with different lanes representing eluted fractions from gel filtration of the $\beta$ FXIIa ${ }^{\text {His }}$-EcTI complex. (b) SPR analysis showing the commercial $\beta$ FXIIa (left) and recombinant $\beta \mathrm{FXIIa}^{\mathrm{His}}$ (right) binding to EcTI. SPR sensorgrams illustrate the $\beta$ FXIIa interactions with immobilized EcTI at various $\beta F X I I a$ concentrations. The arrow indicates increasing $\beta F X I I a$ concentrations used at 0 , $15.625,31.25,62.5,125,250,500,1000 \mathrm{nM}$ with response units (RU) on the y axis. 
(a)

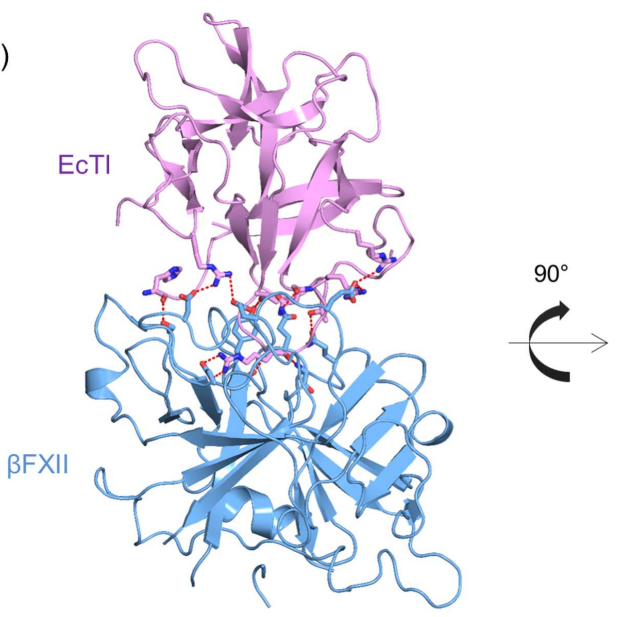

(b)
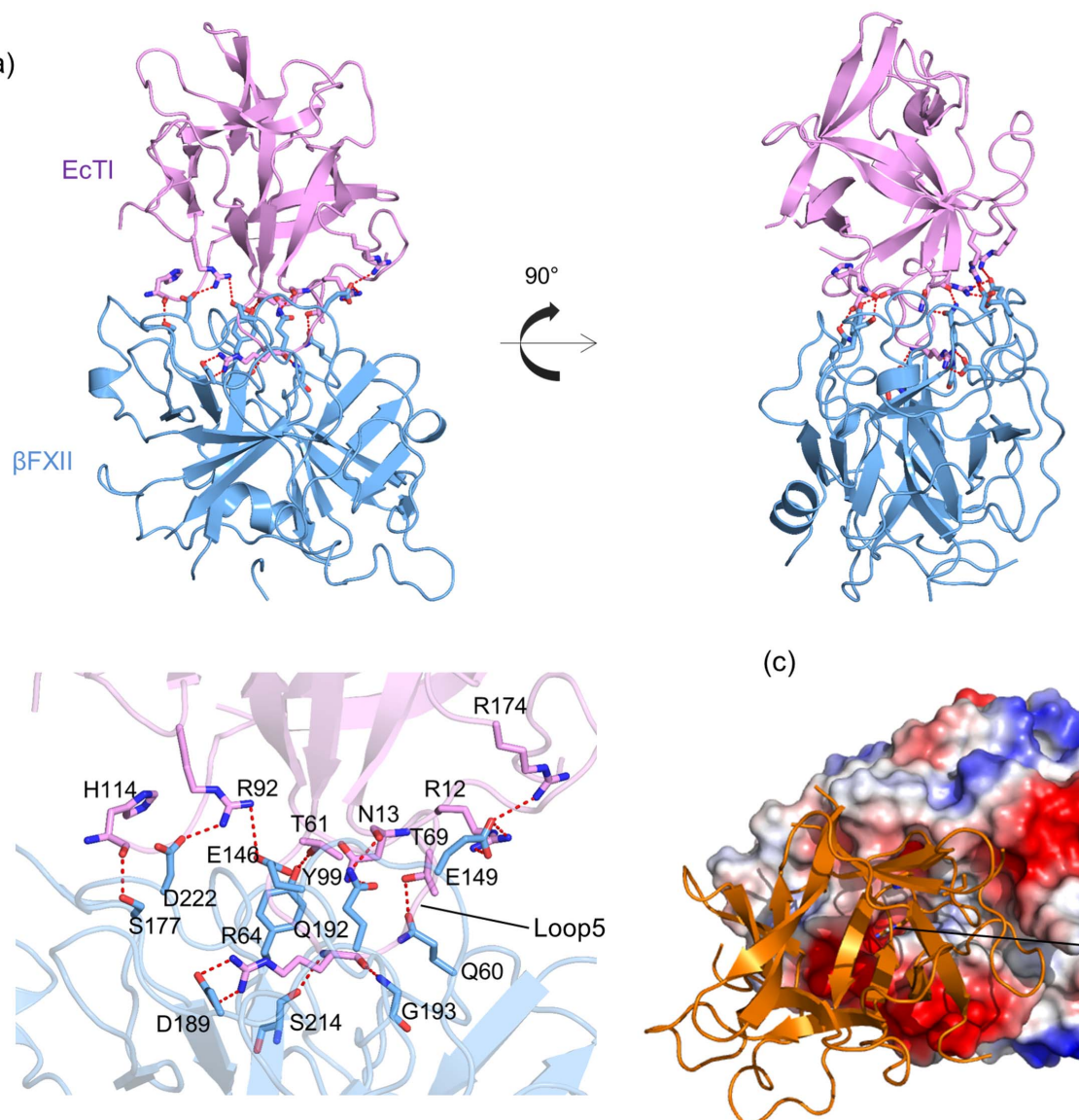

(c)

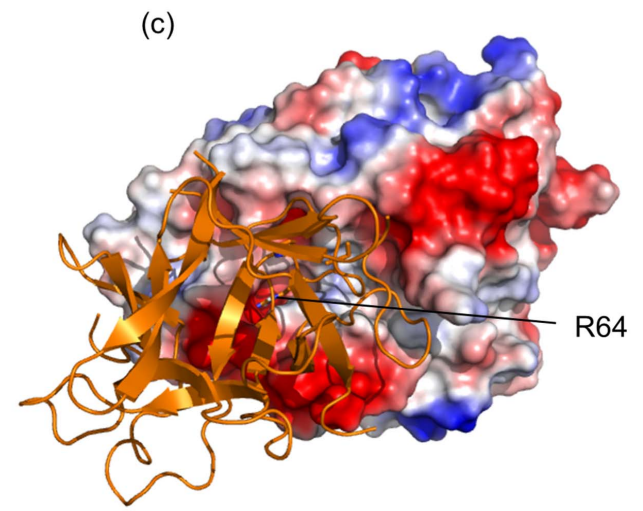

(d)

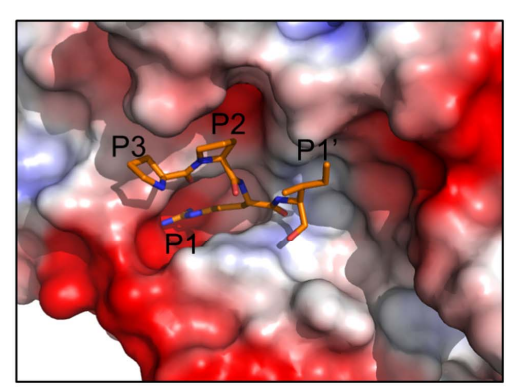

Figure 6. Molecular docking of the inhibitor EcTI with $\beta$ FXIIa ${ }^{\text {His }}$. (a) Cartoon representation of the Cluspro docked complex of $\beta$ FXIIa ${ }^{\text {His }}$ (blue) with EcTI (pink) shown as two rotated views with interfacial residues as sticks. (b) Close up view of the interface between EcTI and $\beta \mathrm{FXIIa}^{\mathrm{His}}$ with the interacting residues drawn in sticks and electrostatic interactions as dashed lines. (c) Charged surface representation of $\beta \mathrm{FXIIa}^{\mathrm{His}}$ with a cartoon representation of the docked EcTI structure (orange). (d) Zoomed in view of the charged surface representation of the active site of $\beta$ FXIIa ${ }^{\text {His }}$ with bound EcTI reactive loop P3-P1' residues shown as sticks (orange). 
(a)
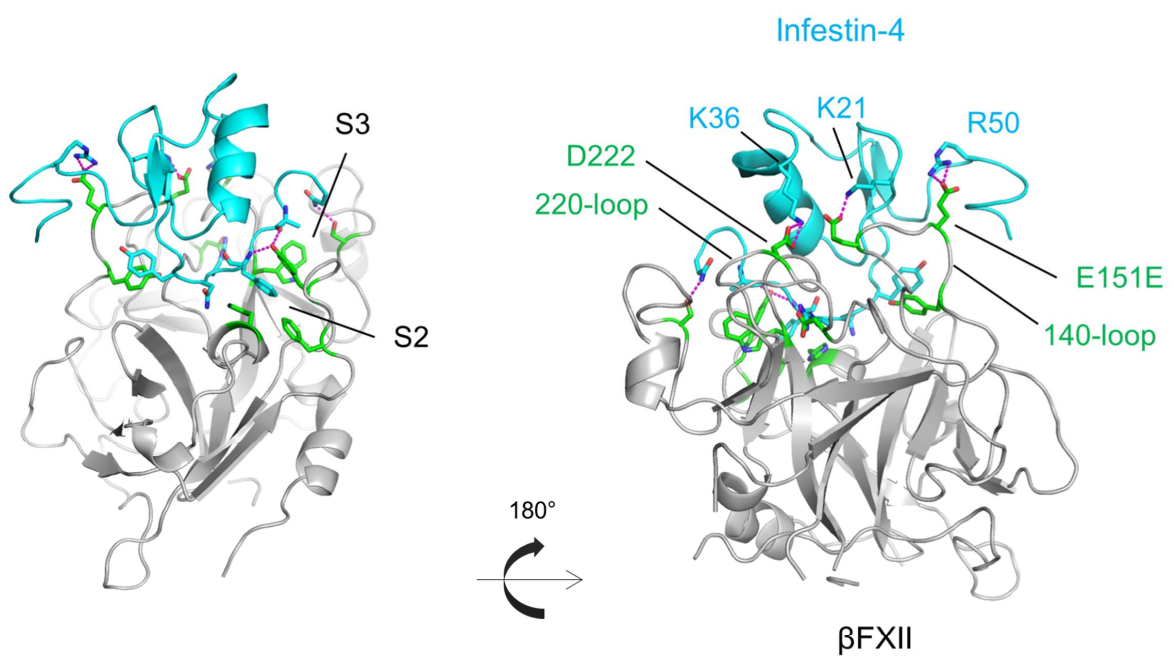

(b)

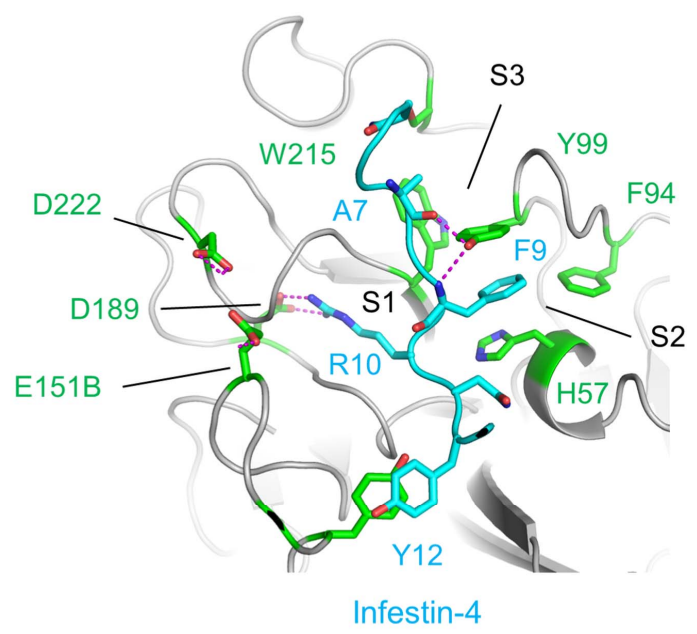

Figure 7. Molecular docking of the Infestin- 4 crystal structure (pdb code:2ERW) with $\beta \mathrm{FXIIa}^{\text {His }}$. (a) Cartoon representation of the Cluspro docked complex of $\beta$ FXIIa ${ }^{\text {His }}$ (grey) with Infestin-4 (cyan) shown as two rotated views with interfacial residues as sticks. (b) Close up view of the interface between Infestin- 4 and $\beta \mathrm{FXIIa}^{\mathrm{His}}$ with the interacting residues drawn in sticks and electrostatic interactions as dashed lines. 
Table 1. Enzyme kinetic parameters for FXIIa samples cleaving substrate S-2302

\begin{tabular}{|c|c|c|c|c|}
\hline Sample & $\begin{array}{l}V_{\max } \\
(\mathrm{pmol} / \mathrm{s})\end{array}$ & $\begin{array}{l}k_{\text {cat }} \\
\left(\mathrm{s}^{-1}\right)\end{array}$ & $\begin{array}{c}K_{m} \\
(\mathbf{m M})\end{array}$ & $\begin{array}{c}k_{\mathrm{cat}} / K_{m} \\
\left(\mathrm{~L} \mathrm{~mol}^{-1} \mathrm{~s}^{-1}\right)\end{array}$ \\
\hline MBP- $\beta$ FXIIa ${ }^{\text {His }}$ & $24.40 \pm 0.60$ & $17.43 \pm 0.83$ & $0.10 \pm 0.02$ & 174300 \\
\hline$\beta \mathrm{FXIIa}^{\mathrm{His}}$ & $32.93 \pm 2.70$ & $21.75 \pm 1.86$ & $0.20 \pm 0.04$ & 108750 \\
\hline$\beta$ FXIIa & $34.20 \pm 3.24$ & $24.44 \pm 0.75$ & $0.16 \pm 0.01$ & 101880 \\
\hline$\alpha$ FXIIa & $21.72 \pm 1.04$ & $15.52 \pm 0.74$ & $0.17 \pm 0.02$ & 91294 \\
\hline
\end{tabular}

Values are reported with standard error of the regression analysis calculated with GRAPHPAD PRISM using a non-linear regression with the Michaelis-Menten equation. 
Table 2. Data collection and refinement statistics

\begin{tabular}{|c|c|c|}
\hline & MBP- $\beta F X I I a{ }^{H i s}$ PPACK & $\beta \mathrm{FXIIa}^{\mathrm{His}}$ \\
\hline \multicolumn{3}{|l|}{ Data Collection } \\
\hline Space group & $\mathrm{P}_{1} 2_{1} 2$ & $\mathrm{P} 4_{1} 2_{1} 2$ \\
\hline \multicolumn{3}{|l|}{ Unit cell dimensions } \\
\hline $\mathrm{a}, \mathrm{b}, \mathrm{c}(\AA)$ & $131.5,131.5,238.4$ & $106.9,106.9,66.0$ \\
\hline$\alpha, \beta, \gamma\left({ }^{\circ}\right)$ & $90,90,90$ & $90,90,90$ \\
\hline Wavelength $(\AA)$ & 0.9795 & 0.97951 \\
\hline Resolution $(\AA)$ & 4.0 & 2.54 \\
\hline $\mathrm{R}_{\text {merge }}$ & $0.189(0.468)$ & $0.218(0.821)$ \\
\hline $\mathrm{I} / \sigma \mathrm{I}$ & $3.8(1.7)$ & $5.6(1.7)$ \\
\hline Completeness (\%) & $94.8(95.7)$ & $99.9(100)$ \\
\hline Multiplicity & 2.5 & 6.3 \\
\hline $\mathrm{CC} 1 / 2$ & $0.95(0.61)$ & $0.985(0.35)$ \\
\hline Unique reflections & $17015(4810)$ & $13131(938)$ \\
\hline \multicolumn{3}{|l|}{ Refinement } \\
\hline $\mathrm{R}_{\text {work }}$ & $0.297(0.368)$ & $0.213(0.219)$ \\
\hline $\mathrm{R}_{\text {free }}$ & $0.356(0.401)$ & $0.265(0.269)$ \\
\hline $\mathrm{B}$-factor $\left(\mathrm{A}^{2}\right)$ & 38.3 & 42.0 \\
\hline \multicolumn{3}{|c|}{ Stereo chemical r.m.s.d. } \\
\hline Bond length $(\AA)$ & 0.011 & 0.010 \\
\hline Bond angle $\left({ }^{\circ}\right)$ & 1.487 & 1.439 \\
\hline \multicolumn{3}{|l|}{ Ramachandran plot } \\
\hline Most favored (\%) & 91.2 & 95.26 \\
\hline Allowed (\%) & 8.8 & 4.74 \\
\hline Outliers (\%) & 0.0 & 0.0 \\
\hline
\end{tabular}


Table 3. Amino acid sequences of FXIla substrate and inhibitor reactive loops.

\begin{tabular}{|c|c|}
\hline & P3P2 P1P1'P2' \\
\hline \multicolumn{2}{|l|}{ Substrates } \\
\hline PK & $\mathrm{S} T \mathrm{~T}$ I $\mathrm{V}$ \\
\hline FXII & $\mathrm{M} \quad \mathrm{T} \quad \mathrm{R} \quad \mathrm{V} \quad \mathrm{V}$ \\
\hline FXI & K $P$ R I V \\
\hline \multicolumn{2}{|l|}{ Inhibitors } \\
\hline PCK & $\mathrm{P} \quad \mathrm{F} R$ \\
\hline Infestin-4 & $\begin{array}{lllll}C & F & R & N\end{array}$ \\
\hline Infestin-4mB & $\mathrm{C} T \mathrm{~T} N \mathrm{~F}$ \\
\hline CTI & G $P \quad \mathbf{R} \quad \mathrm{L} \quad \mathrm{P}$ \\
\hline Bicyclic-pep & $\begin{array}{lllll}C & F & R & P\end{array}$ \\
\hline ECTI & $P \quad P \quad I A$ \\
\hline PPACK & $\begin{array}{lll}F & P & R\end{array}$ \\
\hline
\end{tabular}




\section{References:}

Baglin, T. P., Carrell, R. W., Church, F. C., Esmon, C. T. \& Huntington, J. A. (2002). Proc Natl Acad Sci U S A 99, 11079-11084.

Batista, I. F., Oliva, M. L., Araujo, M. S., Sampaio, M. U., Richardson, M., Fritz, H. \& Sampaio, C. A. (1996). Phytochemistry 41, 1017-1022.

Beinrohr, L., Harmat, V., Dobo, J., Lorincz, Z., Gal, P. \& Zavodszky, P. (2007). J Biol Chem 282, 21100-21109.

Bender, L., Weidmann, H., Rose-John, S., Renne, T. \& Long, A. T. (2017). Front Immunol 8, 1115.

Bode, W., Mayr, I., Baumann, U., Huber, R., Stone, S. R. \& Hofsteenge, J. (1989). Embo j 8, $3467-$ 3475.

Bokhove, M., Sadat Al Hosseini, H., Saito, T., Dioguardi, E., Gegenschatz-Schmid, K., Nishimura, K., Raj, I., de Sanctis, D., Han, L. \& Jovine, L. (2016). J Struct Biol 194, 1-7.

Campos, I. T., Souza, T. A., Torquato, R. J., De Marco, R., Tanaka-Azevedo, A. M., Tanaka, A. S. \& Barbosa, J. A. (2012). Acta Crystallogr D Biol Crystallogr 68, 695-702.

Campos, I. T., Tanaka-Azevedo, A. M. \& Tanaka, A. S. (2004). FEBS Lett 577, 512-516.

Cichon, S., Martin, L., Hennies, H. C., Muller, F., Van Driessche, K., Karpushova, A., Stevens, W., Colombo, R., Renne, T., Drouet, C., Bork, K. \& Nothen, M. M. (2006). Am J Hum Genet 79, 1098-1104.

Dementiev, A., Silva, A., Yee, C., Li, Z., Flavin, M. T., Sham, H. \& Partridge, J. R. (2018). Blood Adv 2, 549-558.

Emsley, P. \& Cowtan, K. (2004). Acta Crystallogr D Biol Crystallogr 60, 2126-2132.

Evans, P. (2006). Acta Crystallogr D Biol Crystallogr 62, 72-82.

Evans, P. R. \& Murshudov, G. N. (2013). Acta Crystallogr D Biol Crystallogr 69, 1204-1214.

Gong, L., Liu, M., Zeng, T., Shi, X., Yuan, C., Andreasen, P. A. \& Huang, M. (2015). J Biol Chem 290, 25795-25804.

Gosalia, D. N., Denney, W. S., Salisbury, C. M., Ellman, J. A. \& Diamond, S. L. (2006). Biotechnol Bioeng 94, 1099-1110.

Hamad, B. K., Pathak, M., Manna, R., Fischer, P. M., Emsley, J. \& Dekker, L. V. (2017). J Thromb Haemost 15, 1818-1828.

Hess, R., Wujak, L., Hesse, C., Sewald, K., Jonigk, D., Warnecke, G., Fieguth, H. G., de Maat, S., Maas, C., Bonella, F., Preissner, K. T., Weiss, B., Schaefer, L., Kuebler, W. M., Markart, P. \& Wygrecka, M. (2017). Thromb Haemost 117, 1896-1907.

Hooley, E., McEwan, P. A. \& Emsley, J. (2007). J Thromb Haemost 5, 2461-2466.

Huntington, J. A. (2011). J Thromb Haemost 9 Suppl 1, 26-34.

Ivanov, I., Matafonov, A., Sun, M. F., Cheng, Q., Dickeson, S. K., Verhamme, I. M., Emsley, J. \& Gailani, D. (2017). Blood 129, 1527-1537.

Iwaki, T. \& Castellino, F. J. (2008). Cytotechnology 57, 45-49.

Jin, T., Chuenchor, W., Jiang, J., Cheng, J., Li, Y., Fang, K., Huang, M., Smith, P. \& Xiao, T. S. (2017). Sci Rep 7, 40991.

Kabsch, W. (2010). Acta Crystallogr D Biol Crystallogr 66, 125-132.

Kleinschnitz, C., Stoll, G., Bendszus, M., Schuh, K., Pauer, H. U., Burfeind, P., Renne, C., Gailani, D., Nieswandt, B. \& Renne, T. (2006). J Exp Med 203, 513-518.

Kolyadko, V. N., Lushchekina, S. V., Vuimo, T. A., Surov, S. S., Ovsepyan, R. A., Korneeva, V. A., Vorobiev, II, Orlova, N. A., Minakhin, L., Kuznedelov, K., Severinov, K. V., Ataullakhanov, F. I. \& Panteleev, M. A. (2015). PLoS One 10, e0144940.

Kozakov, D., Hall, D. R., Xia, B., Porter, K. A., Padhorny, D., Yueh, C., Beglov, D. \& Vajda, S. (2017). Nat Protoc 12, 255-278.

Kristensen, L. H., Olsen, O. H., Blouse, G. E. \& Brandstetter, H. (2016). Biochem J 473, 2395-2411.

Krupka, J., May, F., Weimer, T., Pragst, I., Kleinschnitz, C., Stoll, G., Panousis, C., Dickneite, G. \& Nolte, M. W. (2016). PLoS One 11, e0146783. 
Larsson, M., Rayzman, V., Nolte, M. W., Nickel, K. F., Bjorkqvist, J., Jamsa, A., Hardy, M. P., Fries, M., Schmidbauer, S., Hedenqvist, P., Broome, M., Pragst, I., Dickneite, G., Wilson, M. J., Nash, A. D., Panousis, C. \& Renne, T. (2014). Sci Transl Med 6, 222 ra217.

Maas, C. \& Renne, T. (2018). Blood.

MacQuarrie, J. L., Stafford, A. R., Yau, J. W., Leslie, B. A., Vu, T. T., Fredenburgh, J. C. \& Weitz, J. I. (2011). Blood 117, 4134-4141.

Mahoney, W. C., Hermodson, M. A., Jones, B., Powers, D. D., Corfman, R. S. \& Reeck, G. R. (1984). J Biol Chem 259, 8412-8416.

Matafonov, A., Leung, P. Y., Gailani, A. E., Grach, S. L., Puy, C., Cheng, Q., Sun, M. F., McCarty, O. J., Tucker, E. I., Kataoka, H., Renne, T., Morrissey, J. H., Gruber, A. \& Gailani, D. (2014). Blood 123, 1739-1746.

McCoy, A. J., Grosse-Kunstleve, R. W., Adams, P. D., Winn, M. D., Storoni, L. C. \& Read, R. J. (2007). J Appl Crystallogr 40, 658-674.

Middendorp, S. J., Wilbs, J., Quarroz, C., Calzavarini, S., Angelillo-Scherrer, A. \& Heinis, C. (2017). J Med Chem 60, 1151-1158.

Mohammed, B. M., Matafonov, A., Ivanov, I., Sun, M. F., Cheng, Q., Dickeson, S. K., Li, C., Sun, D., Verhamme, I. M., Emsley, J. \& Gailani, D. (2018). Thromb Res 161, 94-105.

Moon, A. F., Mueller, G. A., Zhong, X. \& Pedersen, L. C. (2010). Protein Sci 19, 901-913.

Murshudov, G. N., Skubak, P., Lebedev, A. A., Pannu, N. S., Steiner, R. A., Nicholls, R. A., Winn, M. D., Long, F. \& Vagin, A. A. (2011). Acta Crystallogr D Biol Crystallogr 67, 355-367.

Nickel, K. F., Long, A. T., Fuchs, T. A., Butler, L. M. \& Renne, T. (2017). Arterioscler Thromb Vasc Biol 37, 13-20.

Pathak, M., Wilmann, P., Awford, J., Li, C., Hamad, B. K., Fischer, P. M., Dreveny, I., Dekker, L. V. \& Emsley, J. (2015). J Thromb Haemost 13, 580-591.

Renne, T., Schmaier, A. H., Nickel, K. F., Blomback, M. \& Maas, C. (2012). Blood 120, 4296-4303.

Reuten, R., Nikodemus, D., Oliveira, M. B., Patel, T. R., Brachvogel, B., Breloy, I., Stetefeld, J. \& Koch, M. (2016). PLoS One 11, e0152386.

Towbin, H., Staehelin, T. \& Gordon, J. (1979). Proc Natl Acad Sci U S A 76, 4350-4354.

Ullah, H., Scappini, E. L., Moon, A. F., Williams, L. V., Armstrong, D. L. \& Pedersen, L. C. (2008). Protein Sci 17, 1771-1780.

Ulmer, J. S., Lindquist, R. N., Dennis, M. S. \& Lazarus, R. A. (1995). FEBS Lett 365, 159-163.

Waugh, D. S. (2016). Postepy Biochem 62, 377-382.

Weidmann, H., Heikaus, L., Long, A. T., Naudin, C., Schluter, H. \& Renne, T. (2017). Biochim Biophys Acta 1864, 2118-2127.

Winter, G., Waterman, D. G., Parkhurst, J. M., Brewster, A. S., Gildea, R. J., Gerstel, M., FuentesMontero, L., Vollmar, M., Michels-Clark, T., Young, I. D., Sauter, N. K. \& Evans, G. (2018). Acta Crystallogr D Struct Biol 74, 85-97.

Wong, S. S., Ostergaard, S., Hall, G., Li, C., Williams, P. M., Stennicke, H. \& Emsley, J. (2016). Blood 127, 2915-2923.

Worm, M., Kohler, E. C., Panda, R., Long, A., Butler, L. M., Stavrou, E. X., Nickel, K. F., Fuchs, T. A. \& Renne, T. (2015). Ann Transl Med 3, 247.

Zhou, D., Lobo, Y. A., Batista, I. F., Marques-Porto, R., Gustchina, A., Oliva, M. L. \& Wlodawer, A. (2013). PLoS One 8, e62252. 
Supporting information for article:

Crystal structures of the recombinant $\beta$-Factor XIIa protease with bound Pro-Arg and Thr-Arg substrate mimetics

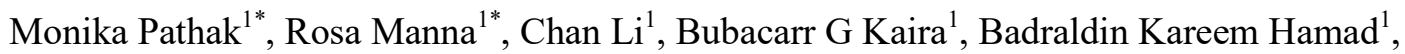
Danilo D Belviso ${ }^{1}$, Camila R Bonturi ${ }^{2}$, Ingrid Dreveny ${ }^{1}$, Peter M Fischer ${ }^{1}$, Lodewijk V Dekker $^{1}$, Maria Luiza V Oliva ${ }^{2}$, and Jonas Emsley ${ }^{1 \uparrow}$. 
(a)

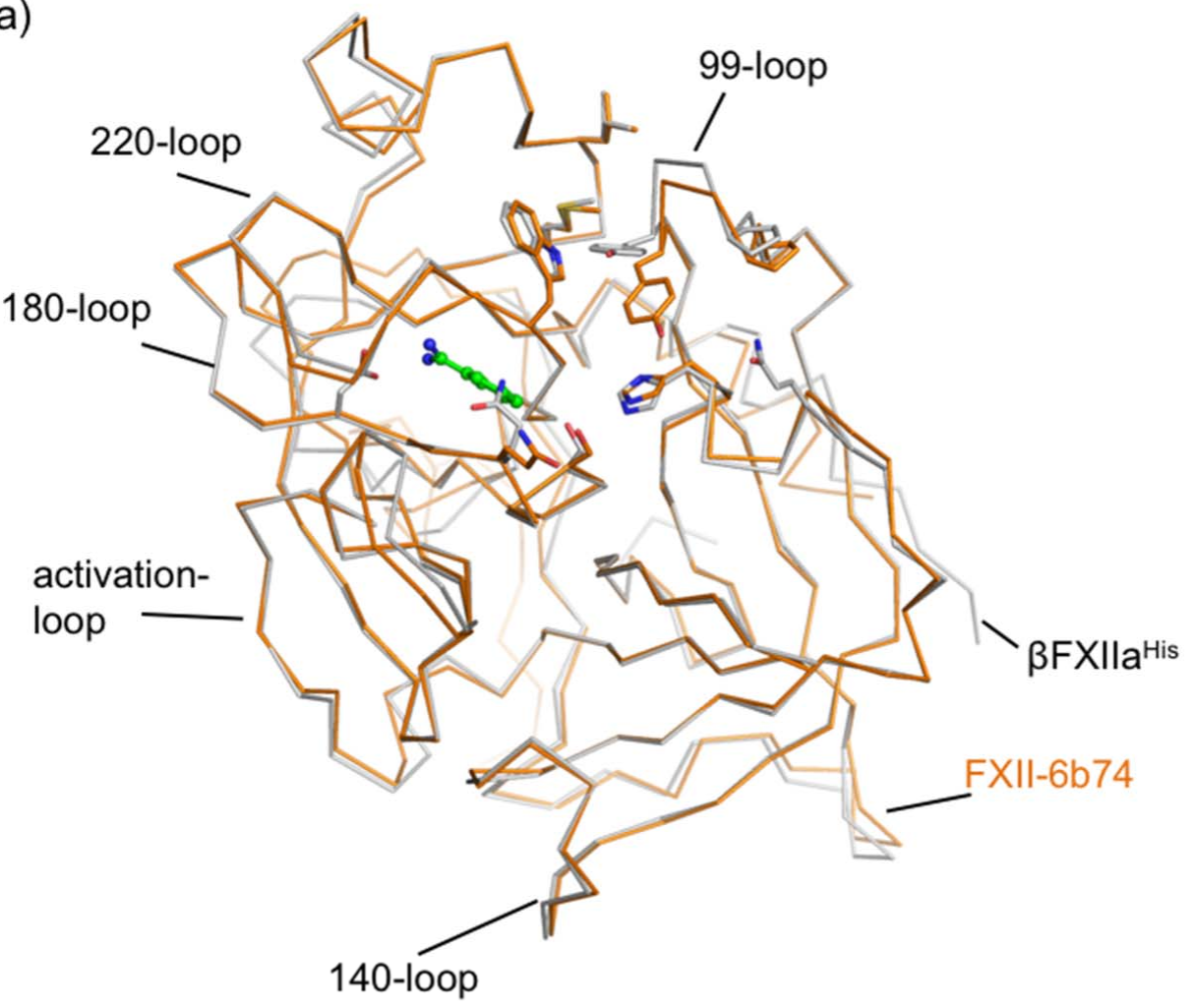

(b)

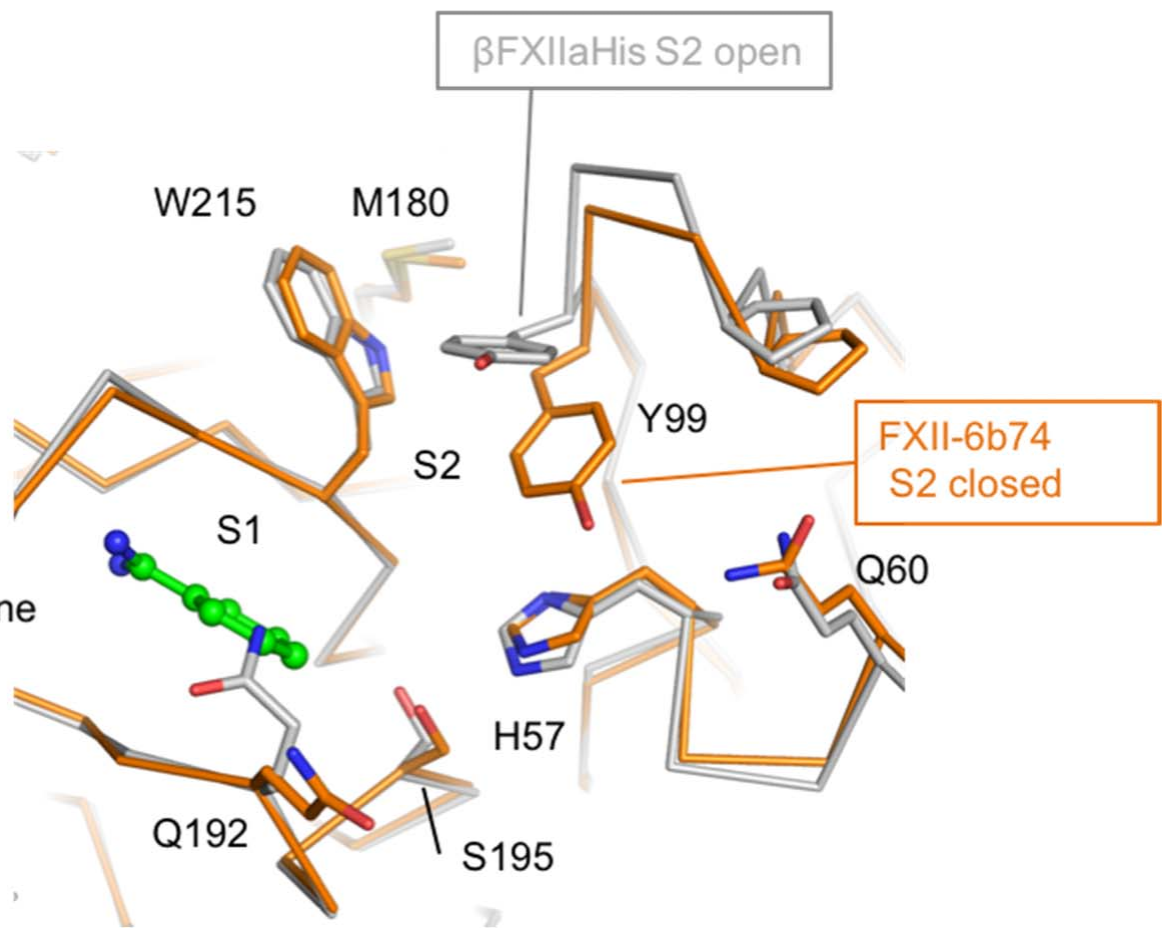

Figure S1. (a) Recombinant and plasma purified $\beta$ FXIIa crystal structures compared. C- $\alpha$ traces are displayed for superposed recombinant protease domain $\beta \mathrm{FXIIa}{ }^{\mathrm{His}}$ (grey) and plasma purified $\beta F X I I a$ (pdb code:6b74, orange) generated using PyMol and overall r.m.s.d. for the superposition $=0.513 \AA$ (b) Zoomed in view illustrating key differences in the region of the 99-loop and the S2 pocket with key residues highlighted as sticks. 

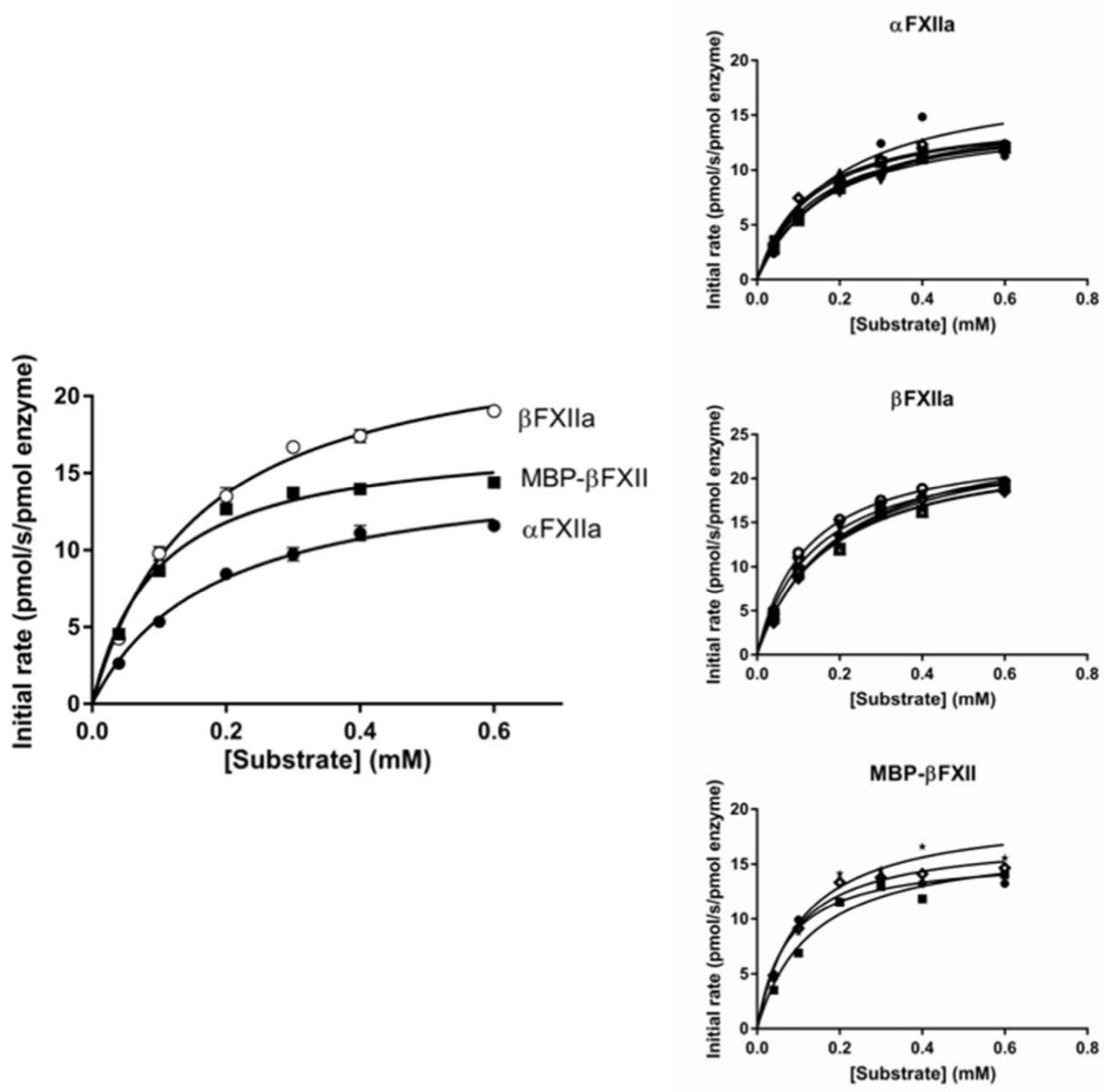

Figure S2. Plot of enzyme substrate S2302 hydrolysis as function of substrate concentration for recombinant MBP- $\beta$ FXIIa ${ }^{\text {His }}$, commercial $\beta$ FXIIa and commercial $\alpha$ FXIIa. OD at $405 \mathrm{~nm}$ (as a function of time ( $\mathrm{min}$ )) for different concentrations of S2302 added to BFXIIa was used to calculate initial rate $\left(\mathrm{pmols}^{-1}\right.$ ) of S2302 hydrolysis (left panel). An average of four experiments were used to determine the enzyme kinetics with independent plots superposed shown on the right panels. 


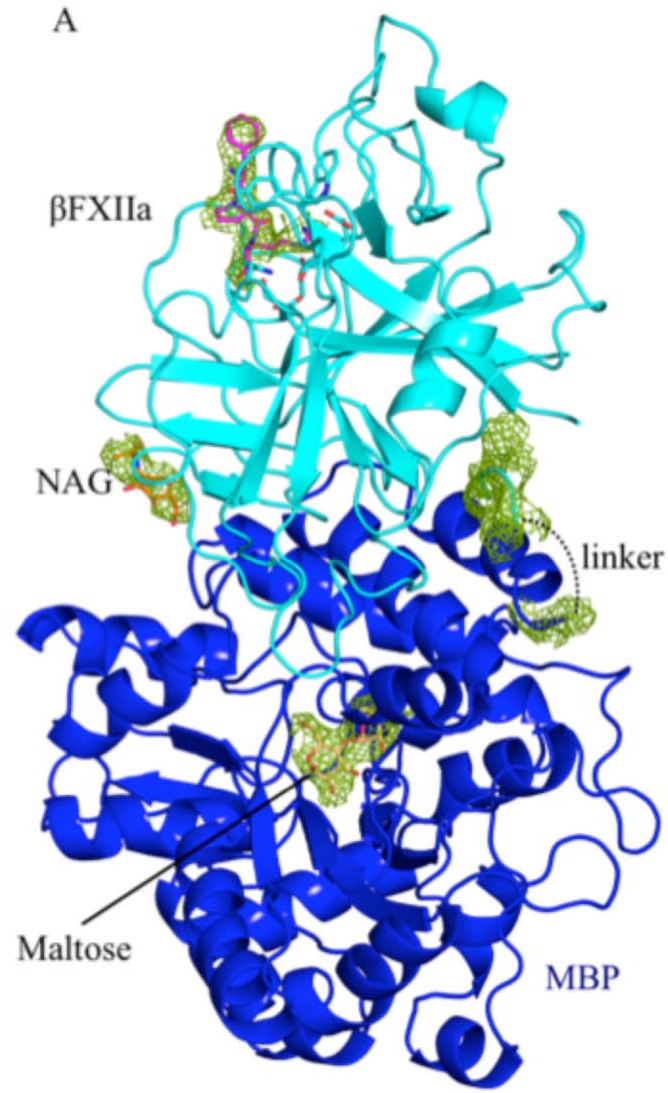

$\mathrm{C}$

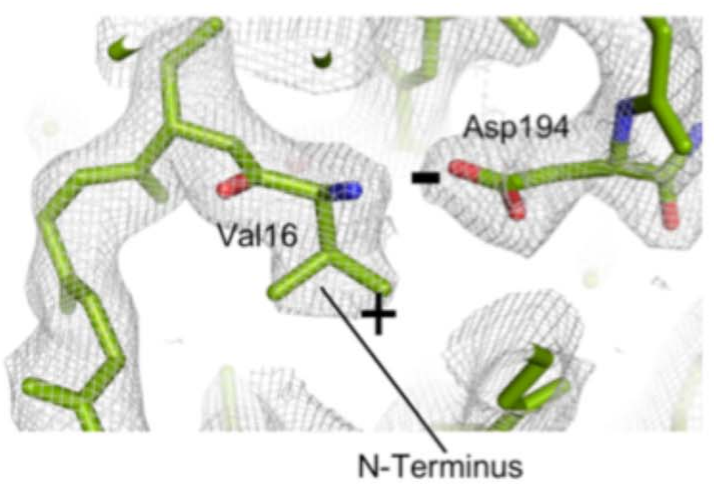

B

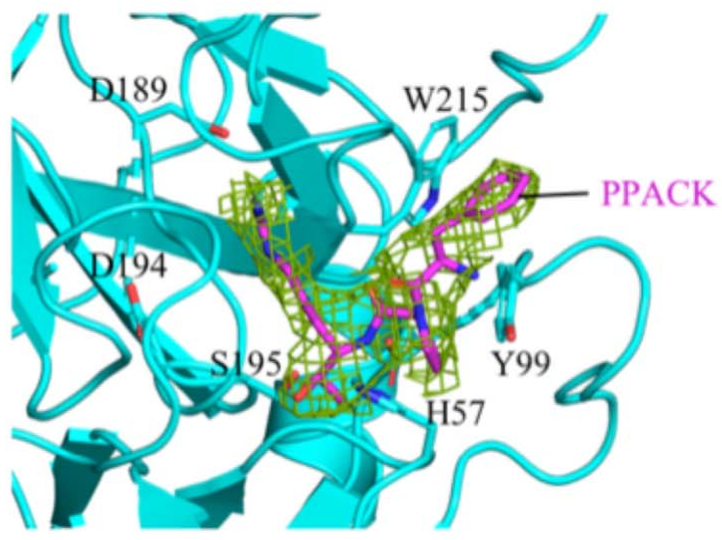

D

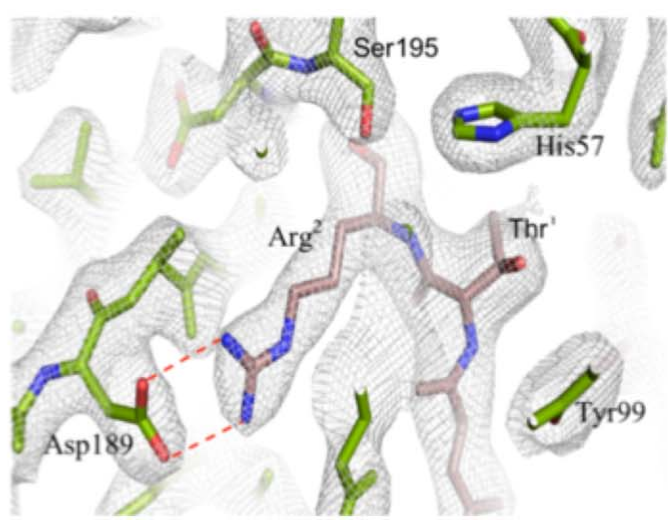

Figure S3. Cartoon diagram of the MBP- $\beta$ FXIIa ${ }^{\text {His }}$ structure. MBP and $\beta$ FXIIa ${ }^{\text {His }}$ are shown in blue and cyan respectively. $A$, Electron density $2 \mathrm{Fo}-\mathrm{Fc}$ maps at $4 \AA$ resolution are contoured at $1.0 \sigma$ are shown (green) for the bound maltose and N-linked glycan. $B$. Electron density Fo-Fc difference map showing the position of PPACK in the $\beta$ FXIIa $^{\text {His }}$ active site cleft. Side chains interacting with PPACK and the catalytic triad are drawn as sticks. $C$, Electron density $2 \mathrm{Fo}-\mathrm{Fc}$ maps at $2.54 \AA$ resolution are shown contoured at $1.0 \sigma$ (grey) for $\beta$ FXIIa $^{\text {His }}$ in the region of the $\mathrm{N}$-terminus and $D$ shows the $\mathrm{S} 1$ pocket with crystal symmetric residue $\mathrm{Arg}^{2}$ (brown) forming electrostatic interactions (red dotted lines). 
Table S1. Summary of the docked EcTI and ßFXIIa interfacial interactions and bond distances.

\begin{tabular}{|c|c|c|c|}
\hline & BFXIIa [atom] & Dist. $[\AA ̊]$ & EcTI [atom] \\
\hline \multicolumn{4}{|c|}{ Hydrogen Bonds } \\
\hline 1 & GLN 192[NE2] & 2.91 & $\mathrm{ASN} 13[\mathrm{O}]$ \\
\hline 2 & TYR 99[OH] & 2.79 & THR 61[ OG1] \\
\hline 3 & GLY $215[\mathrm{~N}]$ & 3.18 & PRO62[ O] \\
\hline 4 & GLY 193[N] & 2.71 & ARG64[ O] \\
\hline 7 & SER 177[OG] & 2.77 & HIS114[ O ] \\
\hline 11 & SER 214[O] & 3.24 & ARG64[N ] \\
\hline 17 & GLN 60[OE1] & 2.83 & THR69[OG1] \\
\hline \multicolumn{4}{|c|}{ Salt Bridges } \\
\hline 1 & GLU 149[OE2] & 3.45 & ARG12[NH1] \\
\hline 2 & GLU 149[OE1] & 2.74 & ARG12[NH1] \\
\hline 3 & GLU 149[OE2] & 2.81 & ARG12[NH2] \\
\hline 4 & GLU 149[OE1] & 2.73 & ARG12[NH2] \\
\hline 5 & ASP 189[OD1] & 2.73 & ARG64[NH1] \\
\hline 6 & ASP 189[OD2] & 3.69 & ARG64[NH1] \\
\hline 7 & ASP 189[OD1] & 3.18 & ARG64[NH2] \\
\hline 8 & ASP 189[OD2] & 2.73 & ARG64[NH2] \\
\hline 9 & ASP 222[OD2] & 3.95 & ARG92[NE ] \\
\hline 10 & ASP 222[OD2] & 2.76 & ARG92[NH1] \\
\hline 11 & GLU 146[OE1] & 3.11 & ARG92[NH1] \\
\hline 12 & GLU 146[OE1] & 3.8 & ARG92[NH2] \\
\hline 13 & GLU 149[OE2] & 3.42 & ARG174[NH1] \\
\hline
\end{tabular}




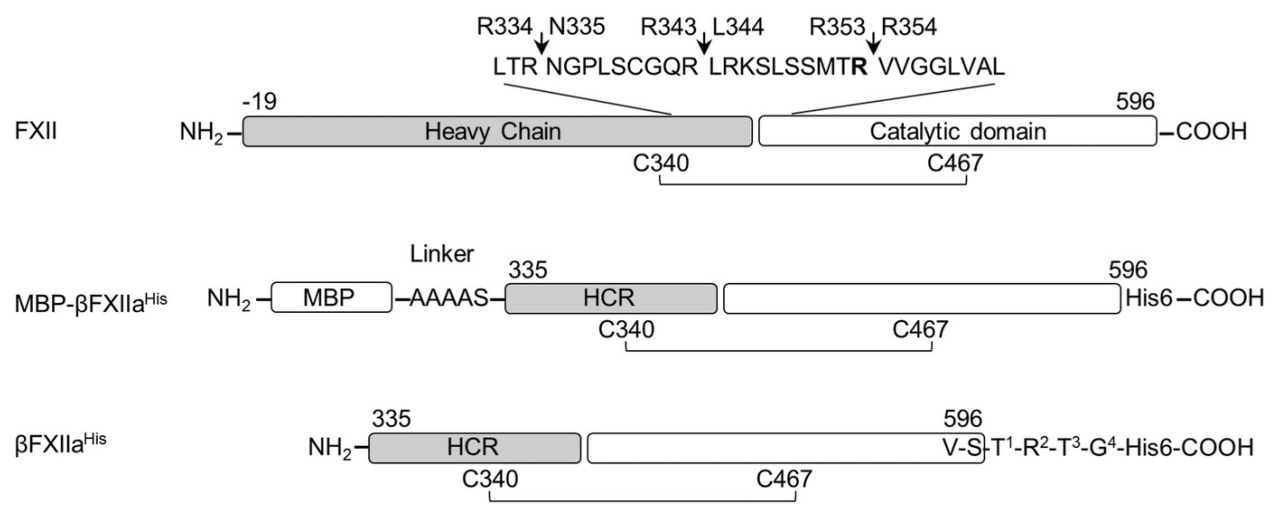

Figure 1 
(a)

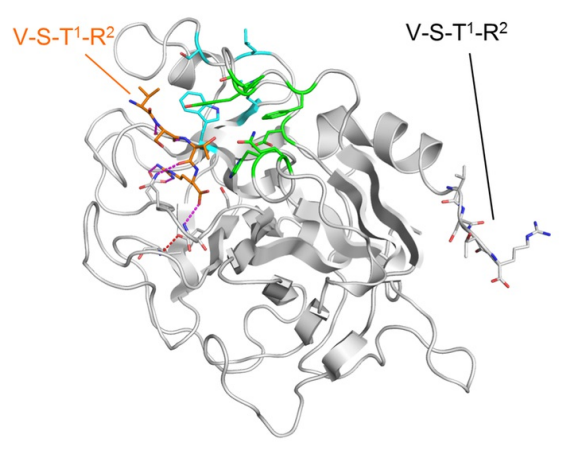

(b)
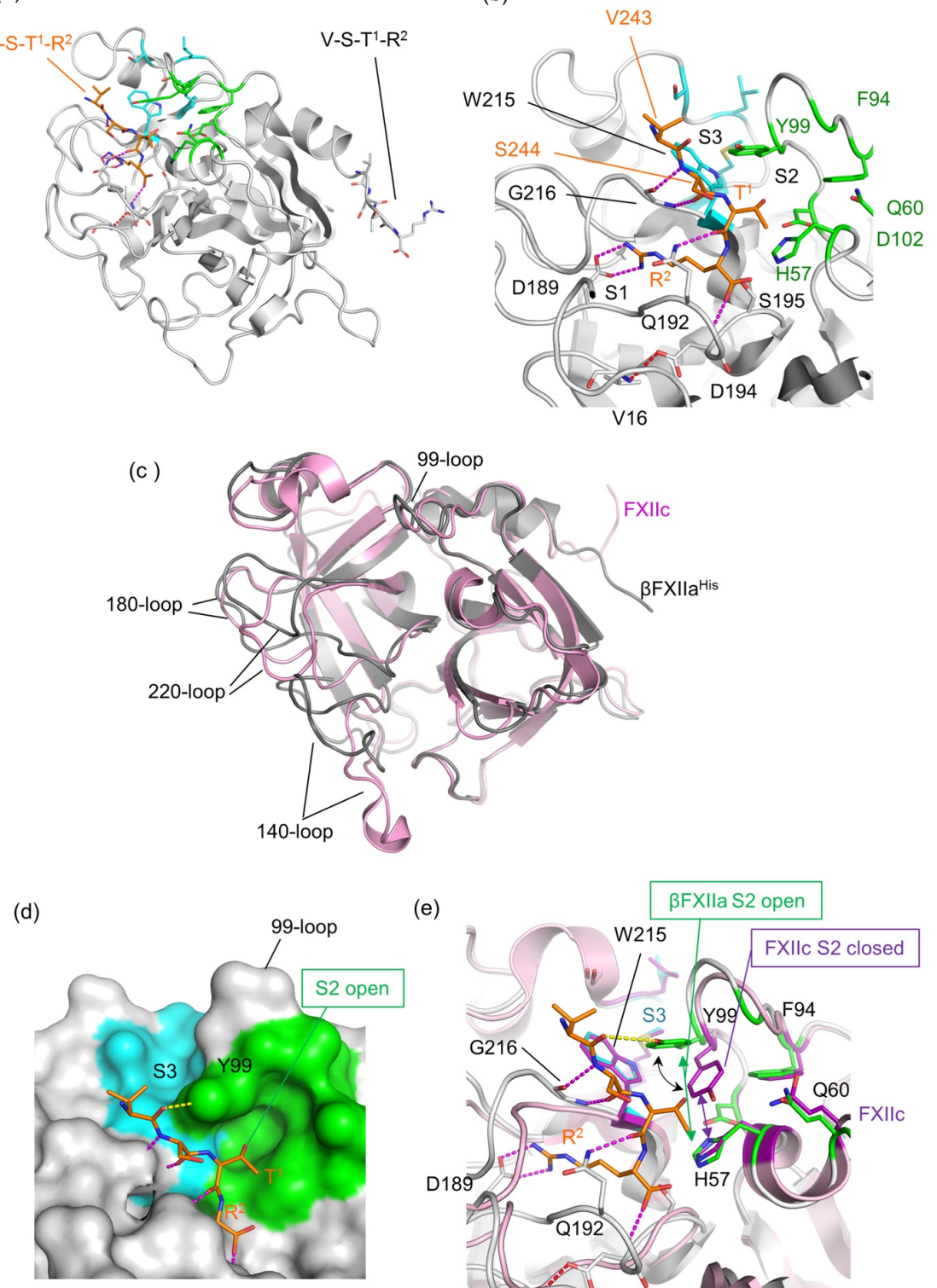

(e)

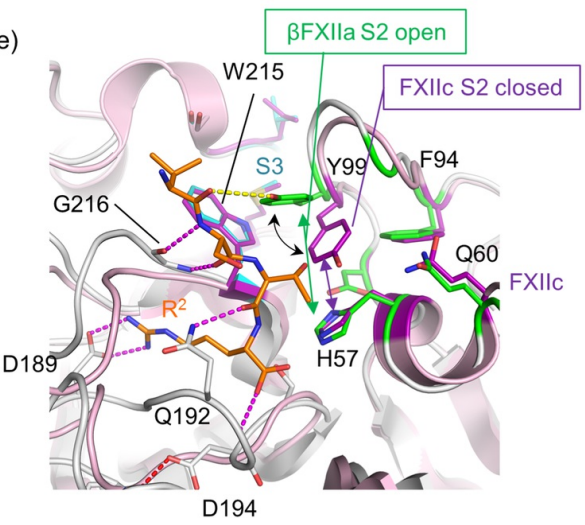

Figure 2 
(a)

FXIIC

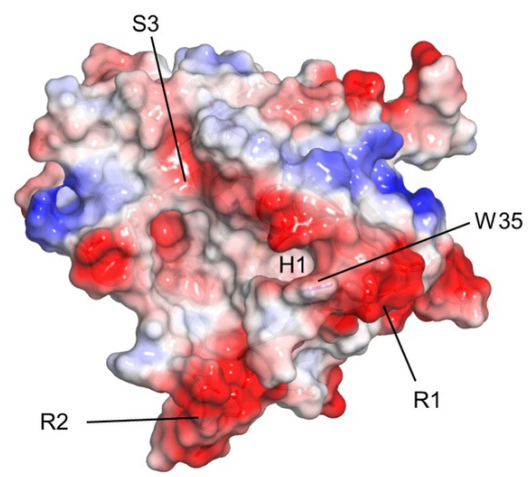

$-63.775-63.775$

(b)

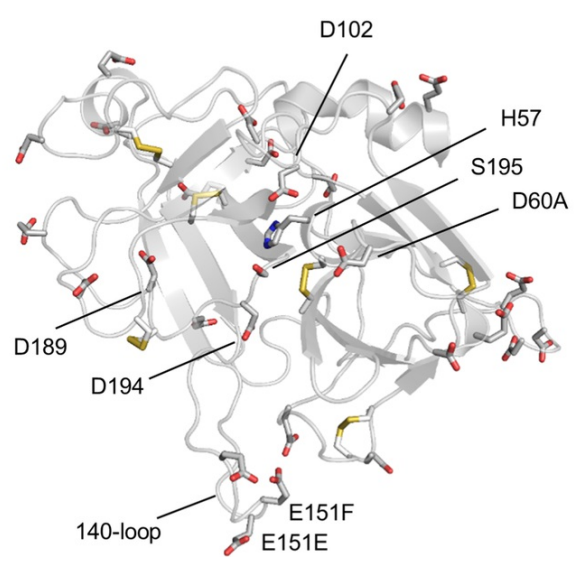

BFXIlaHis

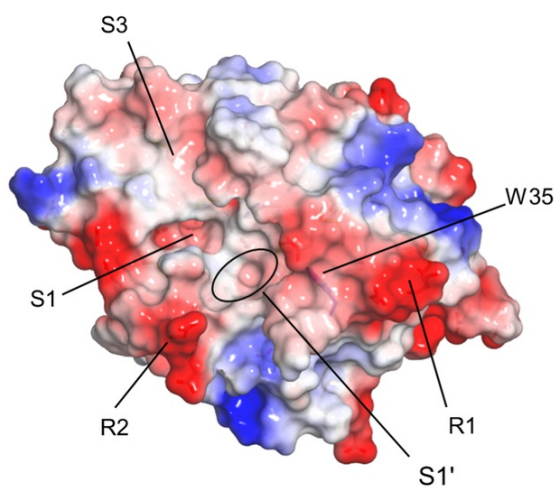

$-125050$

$\beta F X I I a^{H i s}$

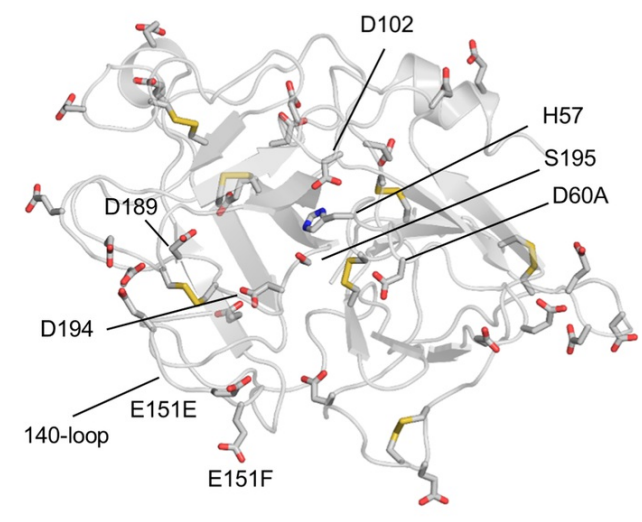

Figure 3 
(a)
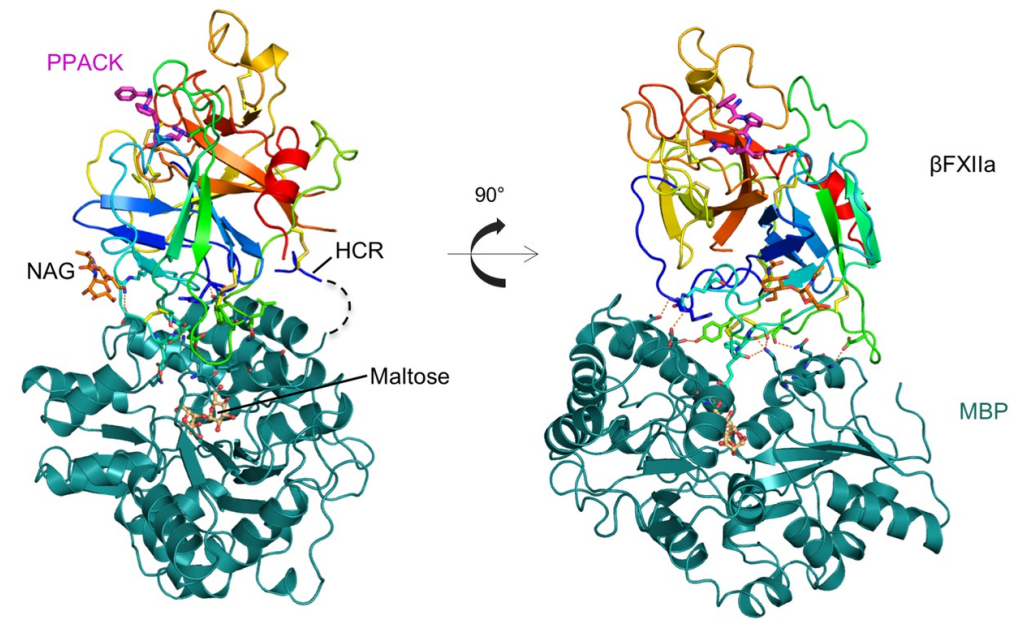

(b)

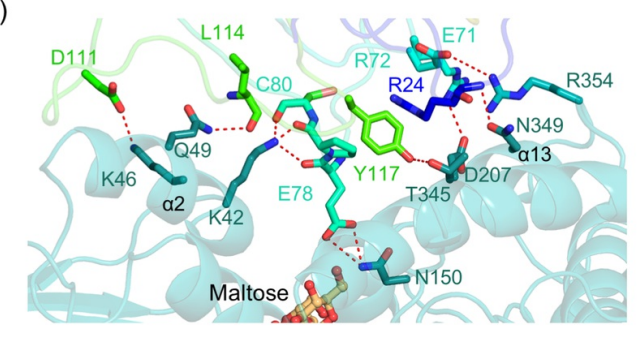

(c)

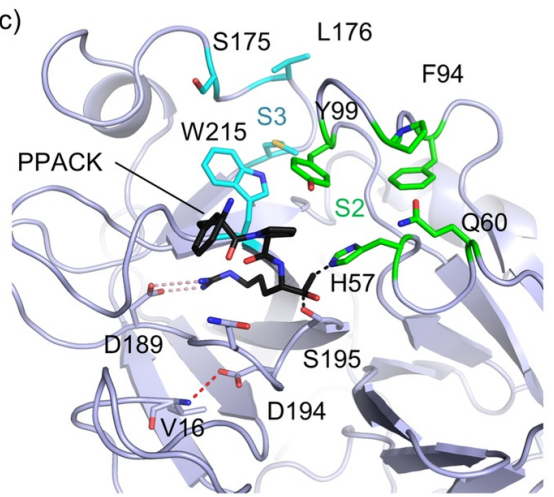

(d)

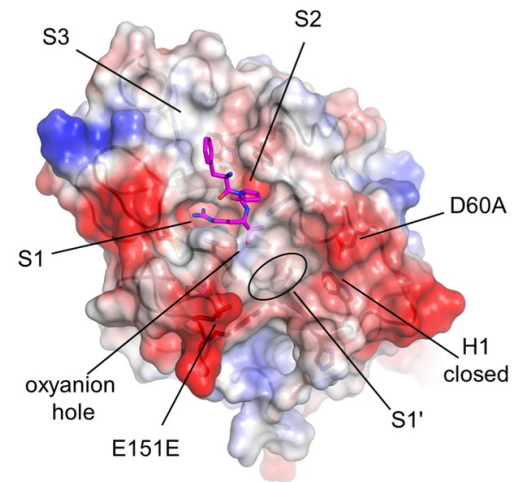

Figure 4 
(a)

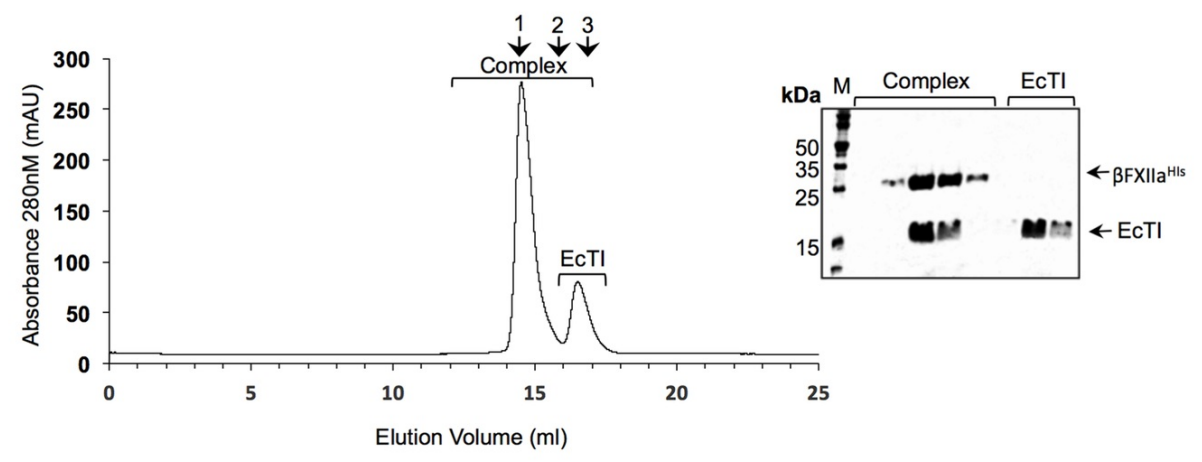

(b)
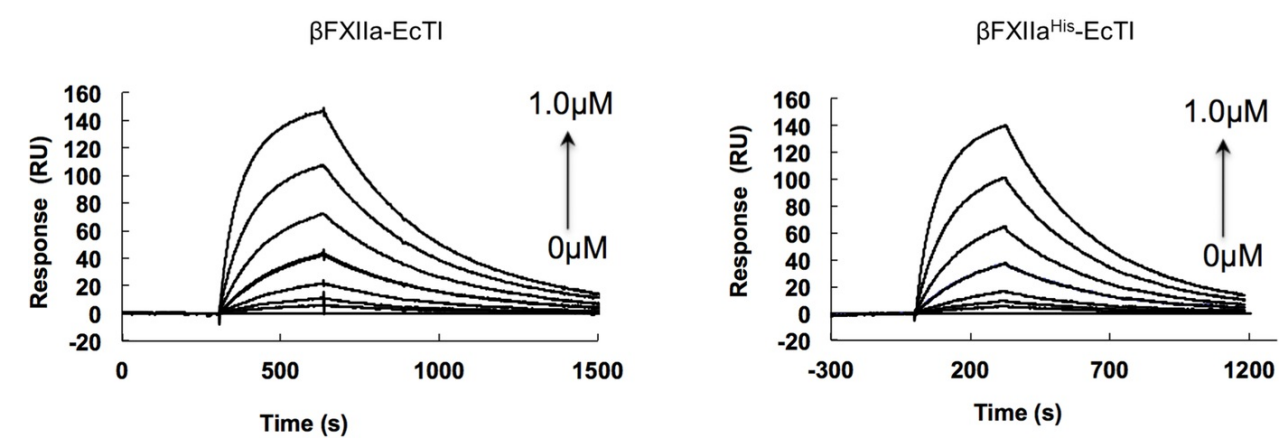

Figure 5 
(a)

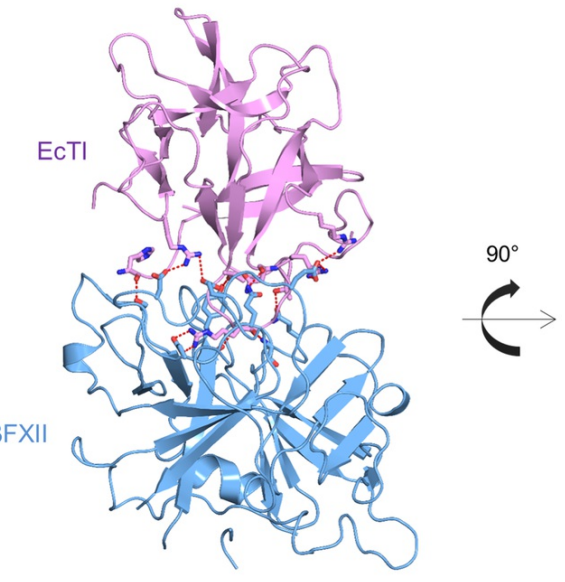

(b)

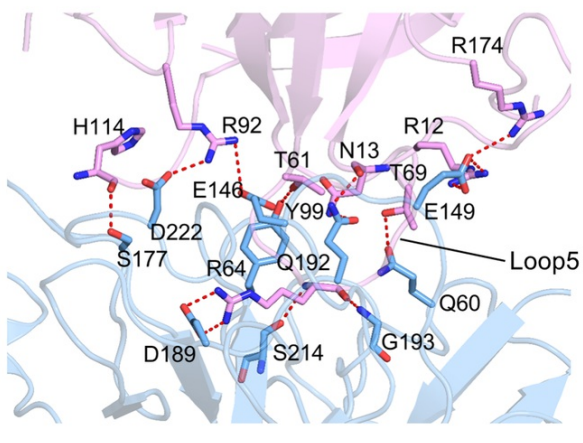

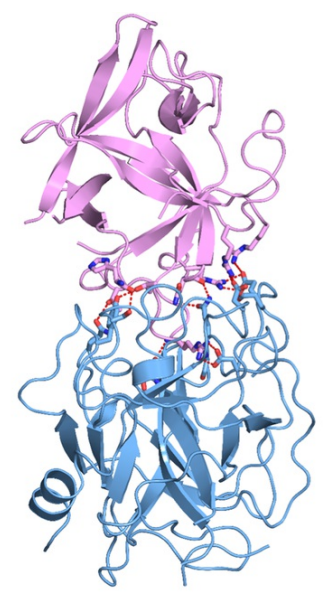

(c)

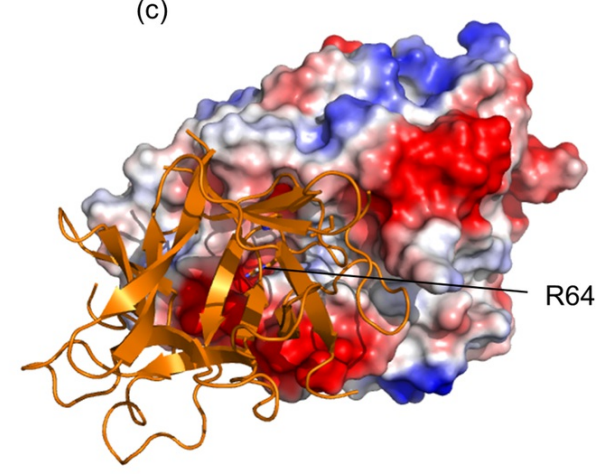

(d)

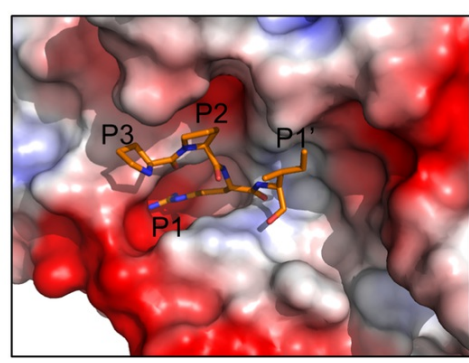

Figure 6 
(a)
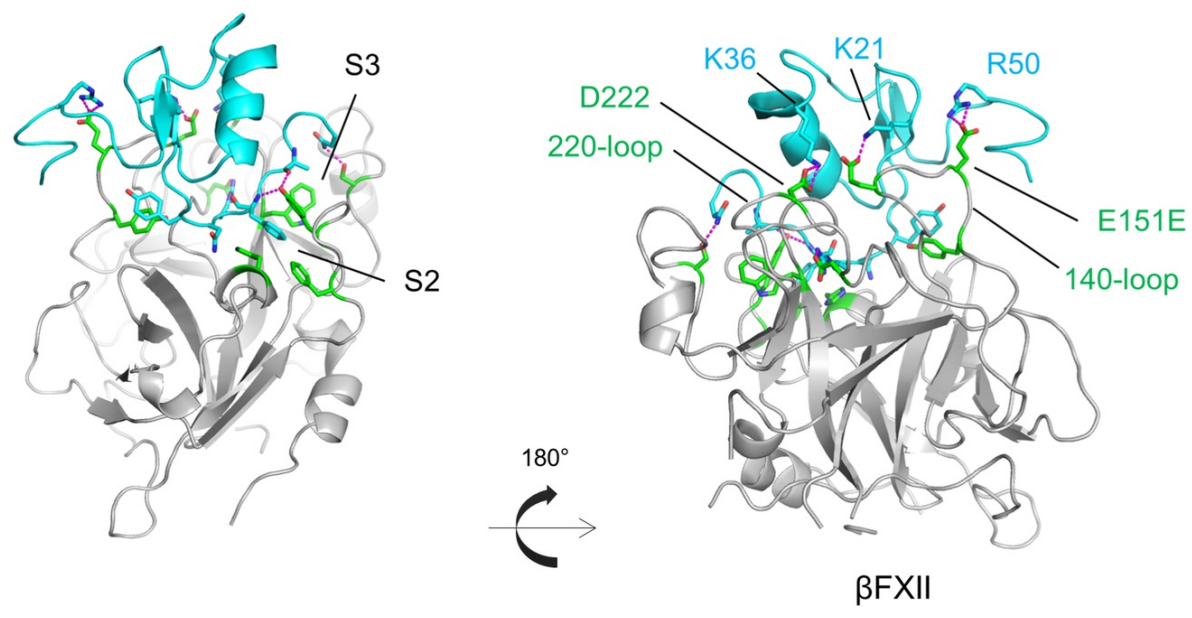

(b)

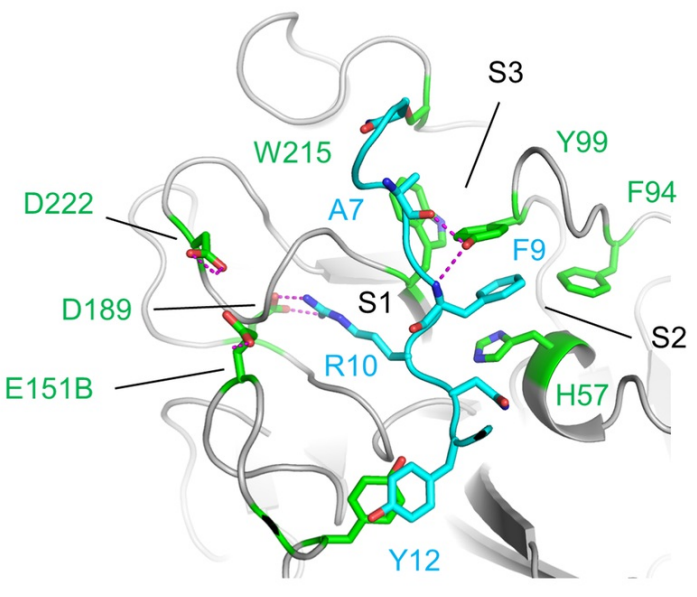

Infestin-4

Figure 7 